\title{
Vertex Algebras in Higher Dimensions and Globally Conformal Invariant Quantum Field Theory
}

\author{
Nikolay M. Nikolov \\ Institute for Nuclear Research and Nuclear Energy \\ Tsarigradsko Chaussee 72, 1784 Sofia, Bulgaria, \\ e-mail:mitov@inrne.bas.bg
}

28th October 2018

\begin{abstract}
We propose an extension of the definition of vertex algebras in arbitrary space-time dimensions together with their basic structure theory. An one-to-one correspondence between these vertex algebras and axiomatic quantum field theory (QFT) with global conformal invariance (GCI) is constructed
\end{abstract}

\section{Contents}

1. Introduction and Notations . . . . . . . . . . . . . . . . . 2

2. Vertex Algebra Definition and Operator Product Expansion . . . . . . . . 7

3. Consequences of the Existence of a Vacuum and of Translation Invariance . 11

4. Existence Theorem. Analytic Continuations . . . . . . . . . . . . . . . 13

5. Free Field Examples. Lie Superalgebras of Formal Distributions . . . . . . 17

6. Categorical Properties of Vertex Algebras. Representations . . . . . . . . 20

7. Conformal Vertex Algebras . . . . . . . . . . . . . . . . . . . . 21

8. Hermitean Structure in Conformal Vertex Algebras . . . . . . . . . . . 25

9. Connection with Globally Conformal Invariant OFT . . . . . . . . . . . 28

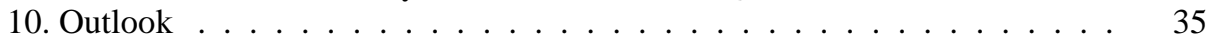




\section{Introduction and Notations}

The axiomatic QFT was proposed and accepted by the physics community about 50 years ago as a collection of mathematically precise structures and their properties which any QFT should posses. Despite the fact that no four dimensional nontrivial model of the axiomatic QFT has been found so far, the long time efforts in this directions have led to several general results such as the Bargmann-Hall-Wightman (BHW) theorem about analytic properties of correlation (i. e. Wightman) functions, the TCP and the spin and statistic theorems. A basic structure in the axiomatic approach is the Poincare symmetry. Right from the beginning the question of extending the space-time symmetry to the conformal one has been posed. It was shown in the article [7] that the condition of GCI, i. e. group conformal invariance, in the frame of the axiomatic QFT leads to the rationality of all correlation functions in any number $D$ of space-time dimensions. This result can be viewed as an extension of the above mentioned BHW theorem. Since the Wightman functions carry the full information of the theory this result shows that the QFT with GCI is essentially algebraic. This gives new insight to the problem of constructing nonfree QFT models in higher dimensions.

In 2 dimensional conformal QFT the theory of vertex algebras is based on simple axiomatic conditions with a straightforward physical interpretation [5]. One of them is the axiom of locality stating that the commutators or anticommutators of the fields vanish when multiplied by a sufficiently large power of the coordinate difference. This axiom has a natural extension to higher dimensions by replacing the coordinate difference with the space-time interval and this is a consequence of GCI in the axiomatic QFT - this is a form of the Huygens principle in QFT called in [7] (see Remark 3.1) strong locality. On the other hand, the rationality of correlation functions in a QFT with GCI allows to define a precise state-field correspondence and an expansion of fields as formal power series in their coordinates $z=\left(z^{1}, \ldots, z^{D}\right)$ and the inverse square interval $\frac{1}{z^{2}}\left(z^{2}=z \cdot z:=\left(z^{1}\right)^{2}+\cdots+\left(z^{D}\right)^{2}\right)$. This provides the second axiomatic structure for the vertex algebras. The coordinates " $z$ " define a chart in the complex compactified Minkowski space containing the entire real compact space and they are useful for connecting the vertex algebra approach with the axiomatic QFT with GCI (see Sect. 9 they are introduced for $D=4$ in [11] and for general $D$, in [8] Sect. 2.2). The existence of the last connection motivates our approach from physical point of view - giving examples of such vertex algebras one would actually obtain models of the Wightman axioms. Physically, one could regard the vertex algebras as providing a realization of the observable field algebra in higher dimensional conformal QFT. The proposed construction of vertex algebras allows to give a precise definition of the notion of their representation which would realize the charged sectors in accord with Haag's program in the algebraic QFT [3].

There is a more general definition of vertex algebras in higher dimensions proposed by Borcherds [1] which allows arbitrary type of singularities occurring in the correlation functions. From the point of view of GCI the only type of singularities arising is the light cone type [7].

The paper is organized as follows.

In Sect. 2 we give the basic definitions and prove the existence of operator product expansions (Theorem 2.1. The vertex algebra fields are denoted by $Y(a, z)$ as in the chiral two-dimensional conformal QFT (chiral CFT), depending on the state $a$ and being formal power series in $z$ including negative powers of $z^{2}$. A convenient basis for such series is provided by the harmonic decomposition of the polynomials in $z$, which 
we will briefly recall bellow. The operator product expansion of two fields $Y(a, z)$ and $Y(b, z)$ is described in terms of infinite series of "products" $Y(a, z)_{\{n, m, \sigma\}} Y(b, z)$ labeled by integers which generalize the analogous products $Y(a, t)_{(n)} Y(b, t)$ in the chiral CFT. The $\{0,0,1\}$-product in our notations is the natural candidate for the normal product in higher-dimensional vertex algebras. In Sect. 3 we obtain an analogue (Theorem 3.1) of the (corollary) of the Reeh-Schlider theorem - the separating property of the vacuum [4]. It is also shown that the state-field correspondence exhausts the class of translation invariant local fields (i. e. the Borchers class, Proposition 3.2). We also obtain generalizations of some basic formulas for the vertex algebras from the chiral CFT. In Sect. 4 we prove a higher dimensional analogue (Theorem 4.1) of the Kac existence theorem ([5], Theorem 4.5) which provides examples of vertex algebras (at least the free ones). In this section we also find a higher dimensional analogue of the associativity identity " $Y(a, z) Y(b, w)=Y(Y(a, z-w) b, w)$ " (Theorem 4.3).

In Sect. [5 we present the free field examples of higher dimensional vertex algebras and also a more general construction based on Lie superalgebras of formal distributions. In Sect. 6 we introduce some constructions with vertex algebras including the basic categorical notions, tensor product and representations of vertex algebras. Sects. 7 and 8 are devoted to the incorporation of the conformal symmetry in higher dimensions and the Hermitean structure (needed for the passage to the GCI QFT) within the vertex algebras.

In Sect. 9 we give an one-to-one correspondence between vertex algebras with additional conformal and Hermitean structure, and the GCI QFT. Thus the free GCI QFT models provide examples for the vertex algebras with additional structure introduced in the previous sections.

Notations. The $z$ - and $w$-variables as $z, z_{1}, z_{2}$, w etc. will always denote $D$ component variables:

$$
z=\left(z^{1}, \ldots, z^{D}\right), \quad z_{k}=\left(z_{k}^{1}, \ldots, z_{k}^{D}\right), \quad w=\left(w^{1}, \ldots, w^{D}\right) .
$$

We fix the standard scalar product:

$$
z_{1} \cdot z_{2}=\sum_{\mu=1}^{D} z_{1}^{\mu} z_{2}^{\mu}, \quad z^{2} \equiv z \cdot z .
$$

$\mathbb{N} \equiv\{1,2, \ldots\}, \mathbb{Z} \equiv\{0, \pm 1, \ldots\}$

For a complex vector space $V, V[z]$ stands for the space of polynomials with coefficients in $V$ (i. e., $V[z] \equiv V \otimes \mathbb{C}[z]$ ). Similarly, $V \llbracket z \rrbracket$ is the space of formal power series in $z$ with coefficients in $V$. We introduce the formal derivatives on $V[z]$ and $V \llbracket z \rrbracket$ :

$$
\partial_{z} \equiv\left(\partial_{z^{1}}, \ldots, \partial_{z^{D}}\right) \equiv\left(\frac{\partial}{\partial z^{1}}, \ldots, \frac{\partial}{\partial z^{D}}\right)
$$

as well as the Euler and Laplace operators:

$$
z \cdot \partial_{z} \equiv \sum_{\mu=1}^{D} z^{\mu} \partial_{z^{\mu}}, \quad \partial_{z}^{2} \equiv \partial_{z} \cdot \partial_{z} \equiv \sum_{\mu=1}^{D}\left(\partial_{z^{\mu}}\right)^{2} .
$$


Then $\partial_{z}$ obeys the Leibniz rule and the homogeneous polynomials of degree $n$ are characterized by the Euler equation $\left(z \cdot \partial_{z} p\right)(z)=n p(z)$. A harmonic polynomial $p(z) \in V[z]$ is such that the Laplace equation $\left(\partial_{z}^{2} p\right)(z)=0$ is satisfied.

The basic fact about the existence of harmonic decomposition can be stated as follows: if $p(z) \in V[z]$ is a homogeneous polynomial of degree $n(\operatorname{deg} p=n)$ then there exists a unique decomposition

$$
p(z)=\sum_{k=0}^{\llbracket \frac{n}{2} \rrbracket}\left(z^{2}\right)^{k} h_{k}(z), \partial_{z}^{2} h_{k}(z)=0, \quad z \cdot \partial_{z} h_{k}(z)=(n-2 k) h_{k}(z),
$$

where $\llbracket a \rrbracket$ stands for the integer part of the real number $a$.

The proof is based on induction in $n=\operatorname{deg} p$ : if $\partial_{z}^{2} p(z)$ has by the inductive assumption a unique decomposition $\partial_{z}^{2} p(z)=\sum_{k=0}^{\llbracket \frac{n}{2} \rrbracket-1}\left(z^{2}\right)^{k} h_{k}^{\prime}(z), \operatorname{deg} h_{k}^{\prime}=n-2-$ $2 k$, then the difference

$$
h_{0}(z):=p(z)-\sum_{k=0}^{\llbracket \frac{n}{2} \rrbracket-1}\left(4(k+1)\left(n-k+\frac{D-4}{2}\right)\right)^{-1}\left(z^{2}\right)^{k+1} h_{k}^{\prime}(z)
$$

is verified to be a harmonic homogeneous polynomial by a straightforward computation. Thus we obtain that $h_{k}(z)=\left(4 k\left(n-k+1+\frac{D-4}{2}\right)\right)^{-1} h_{k-1}^{\prime}(z)$ for $k>0$.

In such a way if we denote by $V_{m}[z]$ the subspace of homogeneous polynomials of $V[z]$ of degree $m$ and by $V_{m}^{\text {harm }}[z]$ the subspace of $V_{m}[z]$ of the harmonic polynomials $\left(V_{m}[z] \equiv V_{m}^{\text {harm }}[z] \equiv\{0\}\right.$ for $\left.m<0\right)$ then we have the decomposition

$$
\begin{aligned}
V_{m}[z] & =V_{m}^{\text {harm }}[z] \oplus z^{2} V_{m-2}[z], \\
\mathfrak{h}_{m}^{D} \equiv \mathfrak{h}_{m}:=\operatorname{dim} \mathbb{C}_{m}^{\text {harm }}[z] & =\operatorname{dim} \mathbb{C}_{m}[z]-\operatorname{dim} \mathbb{C}_{m-2}[z]= \\
& =\left(\begin{array}{c}
m+D-1 \\
D-1
\end{array}\right)-\left(\begin{array}{c}
m+D-3 \\
D-1
\end{array}\right)
\end{aligned}
$$

(recall that $\left.(1-q)^{-D}=\sum_{m=0}^{\infty}\left(\operatorname{dim} \mathbb{C}_{m}[z]\right) q^{m}\right)$. The space $\mathbb{C}_{m}^{\text {harm }}[z]$ carries an irreducible representation of the complex orthogonal group $S O(D ; \mathbb{C})$ for every $m=$ $0,1, \ldots$ Note that $\mathfrak{h}_{0}^{1}=\mathfrak{h}_{1}^{1}=\mathfrak{h}_{0}^{2}=1$ and $\mathfrak{h}_{m+1}^{1}=0, \mathfrak{h}_{m}^{2}=2$ for $m \geqslant 1 ; \mathfrak{h}_{m}^{3}=2 m+1$ and for $D \geqslant 4$ :

$$
\mathfrak{h}_{m-\frac{D}{2}+1}^{D}=\frac{2 m}{(D-2) !}\left(m+\frac{D}{2}-2\right) \ldots\left(m-\frac{D}{2}+2\right),
$$

so that $\mathfrak{h}_{m-\frac{D}{2}+1}^{D}$ is a polynomial in $m$ of degree $D-2$ for $D \geqslant 2$, which is even for $D$ even, and odd for $D$ odd. For $D=4: \mathfrak{h}_{m}=(m+1)^{2}$.

Let us fix for every $m=0,1, \ldots$ a basis in $\mathbb{C}_{m}^{\text {harm }}[z]$ :

$$
\left\{h_{\sigma}^{(m)}(z): \sigma=1, \ldots, \mathfrak{h}_{m}\right\}, h_{1}^{(0)}(z) \equiv 1, h_{\sigma}^{(m)}(z) \equiv 0 \text { iff } m<0 .
$$


Then for every $a(z) \in V \llbracket z \rrbracket$ we have a unique representation:

$$
a(z)=\sum_{n=0}^{\infty} \sum_{m=0}^{\infty} \sum_{\sigma=1}^{\mathfrak{h}_{m}} a_{\{n, m, \sigma\}}\left(z^{2}\right)^{n} h_{\sigma}^{(m)}(z), \quad a_{\{n, m, \sigma\}} \in V .
$$

For every $n, m=0,1, \ldots$ and $\sigma=1, \ldots, \mathfrak{h}_{m}$ there exists a unique homogeneous polynomial $P_{\{n, m, \sigma\}}(z)$ of degree $2 n+m$ such that:

$$
\left.P_{\{n, m, \sigma\}}\left(\partial_{z}\right) a(z)\right|_{z=0}=a_{\{n, m, \sigma\}}
$$

for any $a(z)$ 1.10). In the special case of $m=0\left(\mathfrak{h}_{m}=1\right)$ :

$$
P_{\{n, 0,1\}}(z)=K_{n}\left(z^{2}\right)^{n}
$$

where $K_{n}:=\frac{(D-2) ! !}{2^{n} n !(2 n+D-2) ! !}$ and $k ! !:=k(k-2) \ldots\left(k-2 \llbracket \frac{k}{2} \rrbracket\right)$. In general, $P_{\{n, m, \sigma\}}(z)$ could be proven to be proportional to $\left(z^{2}\right)^{n} h_{\sigma}^{(m)}(z)$ under the additional assumption of orthogonality of $h_{\sigma}^{(m)}$ but we will not need its explicit form.

Denote by $V \llbracket z, 1 / z 2 \rrbracket$ the vector space of all formal series:

$$
a(z)=\sum_{n \in \mathbb{Z}} \sum_{m=0}^{\infty} \sum_{\sigma=1}^{\mathfrak{h}_{m}} a_{\{n, m, \sigma\}}\left(z^{2}\right)^{n} h_{\sigma}^{(m)}(z), \quad a_{\{n, m, \sigma\}} \in V .
$$

The subspace of $V \llbracket z, 1 / z^{2} \rrbracket$ of finite series 1.13 will be denoted by $V\left[z, 1 / z^{2}\right]$; the subspace of formal series (1.13) whose sum in $n$ is bounded from below: $V \llbracket z \rrbracket z^{2}-$ i. e. the localization of $V \llbracket z \rrbracket$ with respect to the multiplicative system $\left\{\left(z^{2}\right)^{n}\right\}_{n \in \mathbb{N}}$. Thus $a(z) \in V \llbracket z \rrbracket_{z^{2}}$ iff $\left(z^{2}\right)^{N} a(z) \in V \llbracket z \rrbracket$ for sufficiently large $N$, which we will briefly write as $N \gg 0$.

The spaces $V \llbracket z \rrbracket$ and $V \llbracket z \rrbracket_{z^{2}}$ are $\mathbb{C} \llbracket z \rrbracket$ and $\mathbb{C} \llbracket z \rrbracket_{z^{2}}$ modules, respectively, with derivations $\left\{\partial_{z^{\mu}}\right\}_{\mu=1, \ldots, D}$. For $V \llbracket z, 1 / z^{2 \rrbracket}$ we have a structure of a $\mathbb{C}\left[z, 1 / z^{2}\right]$ module with derivations $\partial_{z^{\mu}}$. To define this structure we use the isomorphism

$$
\begin{gathered}
V \llbracket z, 1 / z^{2 \rrbracket} \cong \\
\cong\left(V \llbracket z \rrbracket_{z^{2}} \oplus V \llbracket z \rrbracket_{z^{2}}\right) /\left\{(c(z),-J[c(z)]): c(z) \in V\left\langle z, 1 / z^{2}\right\rangle\right\},
\end{gathered}
$$

where we set $V\left\langle z, 1 / z^{2}\right\rangle$ to be the space of all formal series (1.13) with finite sum in $n$ but possibly infinite in $m$ (being thus a $\mathbb{C}\left[z, 1 / z^{2}\right]$-module), and

$$
J[a(z)]:=\left.a(w)\right|_{w=\frac{z}{z^{2}}} \equiv \sum_{n \in \mathbb{Z}} \sum_{m=0}^{\infty} \sum_{\sigma=1}^{\mathfrak{h}_{m}} a_{\{-n-m, m, \sigma\}}\left(z^{2}\right)^{n} h_{\sigma}^{(m)}(z),
$$

for $a(z)$ in Eq. 1.13. The so defined $J$ is an involutive automorphism of $V\left[z, 1 / z^{2}\right]$ $\left(J^{2}=\mathbb{I}\right.$ and $J[f a]=J[f] J[a]$ for $\left.f \in \mathbb{C}\left[z, 1 / z^{2}\right], a \in V\left[z, 1 / z^{2}\right]\right)$. The isomorphism (1.14) is generated by the map

$$
V \llbracket z \rrbracket_{z^{2}} \oplus V \llbracket z \rrbracket_{z^{2}} \ni(a(z), b(z)) \longmapsto a(z)+J[b(z)] \in V \llbracket z, 1 / z^{2} \rrbracket .
$$


Then the actions of $f(z) \in \mathbb{C}\left[z, 1 / z^{2}\right]$ and $\partial_{z^{\mu}}$ on $V \llbracket z, 1 / z^{2} \rrbracket$ are generated, in view of the isomorphism (1.14, by:

$$
\begin{gathered}
f(z)(a(z), b(z)):=(f(z) a(z), J[f(z)] b(z)), \\
\partial_{z^{\mu}}(a(z), b(z)):=\left(\partial_{z^{\mu}} a(z), \delta_{z^{\mu}} b(z)\right), \\
\text { where } \delta_{z^{\mu}}:=J \partial_{z^{\mu}} J \equiv z^{2} \partial_{z^{\mu}}-2 z^{\mu} z \cdot \partial_{z} .
\end{gathered}
$$

The derivations $\left\{\partial_{z^{\mu}}, \delta_{z^{\nu}}\right\}_{\mu, \nu=1, \ldots, D}$ give rise to an action of the (complex) conformal Lie algebra so $(D+2 ; \mathbb{C})$ on $V \llbracket z, 1 / z^{2} \rrbracket$.

To obtain the explicit form of the actions of $\mathbb{C}\left[z, 1 / z^{2}\right]$ and $\partial_{z^{\mu}}$ on $V \llbracket z, 1 / z^{2 \rrbracket}$ let us note first that for a homogeneous harmonic polynomial $h_{m}(z)$ of degree $m\left(h_{m} \in\right.$ $\left.V_{m}^{\text {harm }}[z]\right)$, the polynomials:

$$
\partial_{z^{\mu}} h_{m}(z) \quad \text { and } \quad z^{\mu} h_{m}(z)-\frac{1}{2\left(m+\frac{D}{2}-1\right)} z^{2} \partial_{z^{\mu}} h_{m}(z)
$$

are harmonic and homogeneous of degrees $m-1$ and $m+1$, respectively. Therefore, there exist constants $A_{\mu \sigma_{1} \sigma_{2}}^{(m)}$ and $B_{\mu \sigma_{1} \sigma_{2}}^{(m)}$ such that

$$
\begin{aligned}
\partial_{z^{\mu}} h_{\sigma_{1}}^{(m)}(z)=\sum_{\sigma_{2}=1}^{\mathfrak{h}_{m-1}} A_{\mu \sigma_{1} \sigma_{2}}^{(m)} h_{\sigma_{2}}^{(m-1)}(z), \quad z^{\mu} h_{\sigma_{1}}^{(m)}(z)=\sum_{\sigma_{2}=1}^{\mathfrak{h}_{m+1}} B_{\mu \sigma_{1} \sigma_{2}}^{(m)} h_{\sigma_{2}}^{(m+1)}(z)+ \\
+\frac{1}{2\left(m+\frac{D}{2}-1\right)} z^{2} \sum_{\sigma_{2}=1}^{\mathfrak{h}_{m-1}} A_{\mu \sigma_{1} \sigma_{2}}^{(m)} h_{\sigma_{2}}^{(m-1)}(z)
\end{aligned}
$$

Using this equations one can obtain the explicit form of the actions of $z^{\mu}$ and of the derivations $\partial_{z^{\mu}}$ on a general series $a(z) \in V \llbracket z,{ }^{1} / z^{2} \rrbracket$. The coefficients $A_{\mu \sigma_{1} \sigma_{2}}^{(m)}$ and $B_{\mu \sigma_{1} \sigma_{2}}^{(m)}$ define intertwining operators $A^{(m)}: \mathbb{C}_{1}^{\text {harm }}[z] \otimes \mathbb{C}_{m}^{\text {harm }}[z] \rightarrow \mathbb{C}_{m-1}^{\text {harm }}[z]$ and $B^{(m)}: \mathbb{C}_{1}^{\text {harm }}[z] \otimes \mathbb{C}_{m}^{\text {harm }}[z] \rightarrow \mathbb{C}_{m+1}^{\text {harm }}[z]$ as $S O(D ; \mathbb{C})$ representations.

In the same way one can define the spaces

$$
V\left[z_{1},{ }^{1} / z_{1}^{2} ; \ldots ; z_{n},{ }^{1} / z_{n}^{2}\right], V \llbracket z_{1},{ }^{1} / z_{1}^{2} ; \ldots ; z_{n},{ }^{1} z_{z_{n}^{2}} \rrbracket \text { and } V \llbracket z_{1}, \ldots, z_{n} \rrbracket z_{1}^{2} \ldots z_{n}^{2}
$$

(the last symbol stands for the localization of $V \llbracket z_{1}, \ldots, z_{n} \rrbracket$ with respect to the multiplicative system $\left.\left\{\left(z_{1}^{2} \ldots z_{n}^{2}\right)^{n}\right\}_{n \in \mathbb{N}}\right)$. Note that

$$
\begin{aligned}
V\left[z_{1}, 1 / z_{1}^{2} ; \ldots ; z_{n},{ }^{1} / z_{n}^{2}\right] & =\left(V\left[z_{1}, 1 / z_{1}^{2} ; \ldots ; z_{n-1},{ }^{1} / z_{n-1}^{2}\right]\right)\left[z_{n},{ }^{1} / z_{n}^{2}\right], \\
V \llbracket z_{1},{ }^{1} / z_{1}^{2} ; \ldots ; z_{n},{ }^{1} / z_{n}^{2} \rrbracket & =\left(V \llbracket z_{1}, 1 / z_{1}^{2} ; \ldots ; z_{n-1}, 1 / z_{n-1}^{2} \rrbracket\right) \llbracket z_{n},{ }^{1} / z_{n}^{2} \rrbracket .
\end{aligned}
$$

It is important that the $\mathbb{C} \llbracket z \rrbracket_{z^{2}}$-module $V \llbracket z \rrbracket_{z^{2}}$ has no "zero divisors", i. e. if $f(z) a(z)=0$ for $f(z) \in \mathbb{C} \llbracket z \rrbracket z^{2}$ and $a(z) \in V \llbracket z \rrbracket z^{2}$ then $f(z)=0$ or $a(z)=0$.

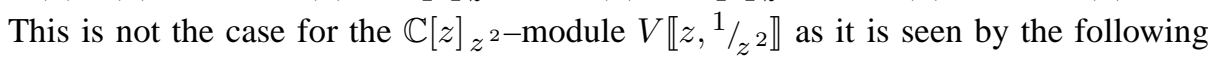
example.

Example 1.1. Let $c=\left(c^{1}, \ldots, c^{D}\right) \in \mathbb{C}^{D}$ be a complex vector such that $c^{2} \equiv c \cdot c$ $=1$. The polynomial $(z-c)^{2}$ is invertible in $\mathbb{C} \llbracket z \rrbracket$ and let $t(z)$ be its inverse. Then $(z-c)^{2}\left(t(z)-\frac{1}{z^{2}} J[t(z)]\right)=0$ and $0 \neq t(z)-\frac{1}{z^{2}} J[t(z)] \in \mathbb{C} \llbracket z, 1 / z^{2} \rrbracket$. 


\section{Vertex Algebra Definition and Operator Product Expansion}

In the next three sections we will use the notation $z_{k l}:=z_{k}-z_{l}$ as an abbreviation of the polynomial $z_{k}-z_{l} \in \mathbb{C}\left[z_{k}, z_{l}\right]$ but not as new variable.

Definition 2.1. Let $\mathcal{V}=\mathcal{V}_{0} \oplus \mathcal{V}_{1}$ be a $\mathbb{Z}_{2}$-graded complex vector space (i. e., a superspace) and $E n d \mathcal{V}=(E n d \mathcal{V})_{0} \oplus(E n d \mathcal{V})_{1}$ be the corresponding Lie superalgebra with bracket $\left[{ }_{-},-\right]$. Then $\mathcal{V}$ is said to be a vertex algebra over $\mathbb{C}^{D}$ if it is equipped with a parity preserving linear map $\mathcal{V} \rightarrow(E n d \mathcal{V}) \llbracket z, 1 / z^{2} \rrbracket: a \mapsto Y(a, z)$, endomorphisms $T_{\mu} \in(E n d \mathcal{V})_{0}$ for $\mu=1, \ldots, D$ called translation endomorphisms and an element $\widehat{1} \in \mathcal{V}_{0}$ called vacuum such that for every $a, a_{1}, a_{2}, b \in \mathcal{V}:$

(a) $\left(z^{2}\right)^{N} Y(a, z) b \in \mathcal{V} \llbracket z \rrbracket$ for $N \gg 0\left(\Leftrightarrow Y(a, z) b \in \mathcal{V} \llbracket z \rrbracket z^{2}\right)$;

(b) $\left(z_{12}^{2}\right)^{N}\left[Y\left(a_{1}, z_{1}\right), Y\left(a_{2}, z_{2}\right)\right]=0$ for $N \gg 0\left(z_{12}:=z_{1}-z_{2}\right)$;

(c) $\left[T_{\mu}, Y(a, z)\right]=\partial_{z^{\mu}} Y(a, z)$ for $\mu=1, \ldots, D$;

(d) $Y(a, z) \hat{1} \in \mathcal{V} \llbracket z \rrbracket$ and $\left.Y(a, z) \hat{1}\right|_{z=0}=a$;

(e) $T_{\mu} \widehat{1}=0$ for $\mu=1, \ldots D ; Y(\widehat{1}, z)=\mathbb{I}$.

The map $a \mapsto Y(a, z)$ is represented as a formal series by:

$$
Y(a, z)=\sum_{n \in \mathbb{Z}} \sum_{m=0}^{\infty} \sum_{\sigma=1}^{\mathfrak{h}_{m}} a_{\{n, m, \sigma\}}\left(z^{2}\right)^{n} h_{\sigma}^{(m)}(z), a_{\{n, m, \sigma\}} \in \text { End } \mathcal{V}
$$

and $Y(a, z) b$ is understood as the series $\sum_{n \in \mathbb{Z}} \sum_{m=0}^{\infty} \sum_{\sigma=1}^{\mathfrak{h}_{m}} a_{\{n, m, \sigma\}} b\left(z^{2}\right)^{n} h_{\sigma}^{(m)}(z) \in$ $\mathcal{V} \llbracket z, 1 / z^{2 \rrbracket}$. For every $a, b \in \mathcal{V}:$

$$
a_{\{n, m, \sigma\}} b=\left.P_{\{n+N, m, \sigma\}}\left(\partial_{z}\right)\left(z^{2}\right)^{N} Y(a, z) b\right|_{z=0} \quad \text { for } \quad N \gg 0
$$

$\left(P_{\{n, m, \sigma\}}(z)\right.$ are defined by Eq. (1.11)). The product $Y\left(a_{1}, z_{1}\right) \ldots Y\left(a_{N}, z_{N}\right)$ will be presented by the series

$$
\begin{aligned}
& Y\left(a_{1}, z_{1}\right) \ldots Y\left(a_{N}, z_{N}\right)= \\
& =\sum_{n_{1} \in \mathbb{Z}} \sum_{m_{1}=0}^{\infty} \sum_{\sigma_{1}=1}^{\mathfrak{h}_{m_{1}}} \ldots \sum_{n_{N} \in \mathbb{Z}} \sum_{m_{N}=0}^{\infty} \sum_{\sigma_{N}=1}^{\mathfrak{h}_{m_{N}}} a_{1\left\{n_{1}, m_{1}, \sigma_{1}\right\}} \ldots a_{N\left\{n_{N}, m_{N}, \sigma_{N}\right\}} \times \\
& \times\left(z_{1}^{2}\right)^{n_{1}} \ldots\left(z_{N}^{2}\right)^{n_{N}} h_{\sigma_{1}}^{\left(m_{1}\right)}\left(z_{1}\right) \ldots h_{\sigma_{N}}^{\left(m_{N}\right)}\left(z_{N}\right)
\end{aligned}
$$

Definition 2.2. Let $\mathcal{V}$ be a superspace. An element

$$
u\left(z_{1}, \ldots, z_{n}\right) \in(\text { End } \mathcal{V}) \llbracket z_{1}, 1 / z_{1}^{2} ; \ldots ; z_{n}, 1 / z_{n}^{2} \rrbracket
$$

is said to be a field if for every $a \in \mathcal{V}: u\left(z_{1}, \ldots, z_{n}\right) a \in \mathcal{V} \llbracket z_{1}, \ldots, z_{n} \rrbracket_{z_{1}^{2} \ldots z_{n}^{2}}$ (i. e., if $\left(z_{1}^{2} \ldots z_{n}^{2}\right)^{N_{a}} u\left(z_{1}, \ldots, z_{n}\right) a \in \mathcal{V} \llbracket z_{1}, \ldots, z_{n} \rrbracket$ for $\left.N_{a} \gg 0\right)$. 
Thus in the case of a vertex algebra $\mathcal{V}, Y(a, z)$ are fields for every $a \in \mathcal{V}$, in accord with Definition 2.1 (a). If $u\left(z_{1}, \ldots, z_{n}\right)$ is a field then we can define $u(z, \ldots, z)$ by setting:

$$
u(z, \ldots, z) a:=\frac{1}{\left(z^{2}\right)^{n N_{a}}}\left[\left.\left(z_{1}^{2} \ldots z_{n}^{2}\right)^{N_{a}} u\left(z_{1}, \ldots, z_{n}\right) a\right|_{z_{1}=\cdots=z_{n}=z}\right],
$$

for $a \in \mathcal{V}$ and $N_{a} \gg 0$, which does not depend on $N_{a} \in \mathbb{N}$. Clearly, if $u\left(z_{1}, \ldots, z_{n}\right)$ is a field then $u(z, \ldots, z)$ and $\partial_{z_{k}^{\mu}} u\left(z_{1}, \ldots, z_{n}\right)$ are fields too.

Definition 2.3. Let $a(z)$ and $b(z)$ be two fields on a superspace $\mathcal{V}, a^{(0)}(z), b^{(0)}(z)$ and $a^{(1)}(z), b^{(1)}(z)$ be their even and odd parts, respectively (i. e. if $a(z)$ has an expansion of type (1.13) with $a_{\{n, m, \sigma\}} \in \operatorname{End} \mathcal{V}$ then $a^{(0,1)}(z)$ is the formal series with coefficients $a_{\{n, m, \sigma\}}^{(0,1)} \in(E n d \mathcal{V})_{0,1}, a_{\{n, m, \sigma\}}=a_{\{n, m, \sigma\}}^{(0)}+a_{\{n, m, \sigma\}}^{(1)}$ ). The fields $a(z)$ and $b(z)$ are said to be mutually local if $\left(z_{12}^{2}\right)^{N}\left[a^{\left(\varepsilon_{1}\right)}\left(z_{1}\right), b^{\left(\varepsilon_{2}\right)}\left(z_{2}\right)\right]=0$ for $N \gg 0$ and $\varepsilon_{1}, \varepsilon_{2}=0,1$.

Then $\left(z_{12}^{2}\right)^{N} a\left(z_{1}\right) b\left(z_{2}\right)$ is a field for $N \gg 0$. Indeed, if $N \gg 0$ then for all $v \in \mathcal{V}:\left(z_{12}^{2}\right)^{N} a\left(z_{1}\right) b\left(z_{2}\right) v=\left(z_{12}^{2}\right)^{N}\left[b^{(0)}\left(z_{2}\right) a^{(0)}\left(z_{1}\right)+b^{(0)}\left(z_{2}\right) a^{(1)}\left(z_{1}\right)+\right.$ $\left.b^{(1)}\left(z_{2}\right) a^{(0)}\left(z_{1}\right)-b^{(1)}\left(z_{2}\right) a^{(1)}\left(z_{1}\right)\right] v$, in accord with locality, so that for $M \gg 0$ : $\left(z_{1}^{2} z_{2}^{2}\right)^{M}\left(z_{12}^{2}\right)^{N} a\left(z_{1}\right) b\left(z_{2}\right) v \in\left(\mathcal{V} \llbracket z_{1} \rrbracket z_{1}^{2}\right) \llbracket z_{2} \rrbracket \cap\left(\mathcal{V} \llbracket z_{2} \rrbracket z_{2}^{2}\right) \llbracket z_{1} \rrbracket \equiv \mathcal{V} \llbracket z_{1}, z_{2} \rrbracket$.

Theorem 2.1. Let a $(z)$ and $b(z)$ be mutually local fields on a superspace $\mathcal{V}$. Then for $N \gg 0$ and every $M \in \mathbb{N}, v \in \mathcal{V}$, there exists a unique decomposition:

$$
\begin{aligned}
\left(z_{12}^{2}\right)^{N} a\left(z_{1}\right) b\left(z_{2}\right) v= & \sum_{\substack{n, m=0,1, \ldots ; \\
2 n+m \leqslant M}} \sum_{\sigma=1}^{\mathfrak{h}_{m}} \theta_{\{n, m, \sigma\}}^{(N, M)}\left(z_{2}\right) v\left(z_{12}^{2}\right)^{n} h_{\sigma}^{(m)}\left(z_{12}\right)+ \\
& +\sum_{\mu_{1}, \ldots, \mu_{M+1}=1}^{D} z_{12}^{\mu_{1}} \ldots z_{12}^{\mu_{M+1}} \psi_{\mu_{1} \ldots \mu_{M+1}}^{(N, M)}\left(z_{1}, z_{2}\right) v,
\end{aligned}
$$

where $\theta_{\{n, m, \sigma\}}^{(N, M)}(z)$ and $\psi_{\mu_{1} \ldots \mu_{M+1}}^{(N, M)}\left(z_{1}, z_{2}\right)$ are fields. The fields a $(z)_{\{n, m, \sigma\}} b(z):=$ $\theta_{\{n+N, m, \sigma\}}^{(N, M)}(z)$ do not depend on $N$ and $M$ and are determined by

$$
a(z)_{\{n, m, \sigma\}} b(z) v=\left.P_{\{n+N, m, \sigma\}}\left(\partial_{z_{1}}\right)\left(z_{12}^{2}\right)^{N} a\left(z_{1}\right) b\left(z_{2}\right) v\right|_{z_{1}=z_{2}=z}
$$

for sufficiently large $N$, independent on $v \in \mathcal{V}, n \in \mathbb{Z}, m=0,1, \ldots$ and $\sigma=$ $1, \ldots, \mathfrak{h}_{m}\left(P_{\{n+N, m, \sigma\}}(z)\right.$ are the polynomials introduced by (1.11)). If $c(z)$ is another field which is local with respect to $a(z)$ and $b(z)$ then every field $a(z)_{\{n, m, \sigma\}} b(z)$ is also local with respect to $c(z)$.

We will prove first two lemmas. 
Lemma 2.2. Let $f\left(z_{1}, z_{2}\right) \in \mathcal{V} \llbracket z_{1}, z_{2} \rrbracket z_{1}^{2} z_{2}^{2}$. Then for every $M \in \mathbb{N}$ there exists a unique decomposition

$$
\begin{aligned}
f\left(z_{1}, z_{2}\right)= & \sum_{\substack{n, m=0,1, \ldots ; \\
2 n+m \leqslant M}} \sum_{\sigma=1}^{\mathfrak{h}_{m}} g_{\{n, m, \sigma\}}\left(z_{2}\right)\left(z_{12}^{2}\right)^{n} h_{\sigma}^{(m)}\left(z_{12}\right)+ \\
& +\sum_{\mu_{1}, \ldots, \mu_{M+1}=1}^{D} z_{12}^{\mu_{1}} \ldots z_{12}^{\mu_{M+1}} g_{\mu_{1} \ldots \mu_{M+1}}^{(M)}\left(z_{1}, z_{2}\right)
\end{aligned}
$$

where $g_{\{n, m, \sigma\}}(z) \in \mathcal{V} \llbracket z \rrbracket_{z^{2}}$ and $g_{\mu_{1} \ldots \mu_{M+1}}^{(M)}\left(z_{1}, z_{2}\right) \in \mathcal{V} \llbracket z_{1}, z_{2} \rrbracket_{z_{1}^{2} z_{2}^{2}}$. Moreover, if $f\left(z_{1}, z_{2}\right) \in \mathcal{V} \llbracket z_{1}, z_{2} \rrbracket$ then $g_{\{n, m, \sigma\}}(z) \in \mathcal{V} \llbracket z \rrbracket$ and $g_{\mu_{1} \ldots \mu_{M+1}}^{(M)}\left(z_{1}, z_{2}\right) \in \mathcal{V} \llbracket z_{1}, z_{2} \rrbracket$.

Proof. The uniqueness of the decomposition (2.6) follows from the equality

$$
g_{\{n, m, \sigma\}}(z)=\left.P_{\{n, m, \sigma\}}\left(\partial_{z_{1}}\right) f\left(z_{1}, z_{2}\right)\right|_{z_{1}=z_{2}=z}
$$

in accord with Eq. 1.11, so that if $f\left(z_{1}, z_{2}\right) \in \mathcal{V} \llbracket z_{1}, z_{2} \rrbracket$ then $\forall g_{\{n, m, \sigma\}}(z) \in$ $\mathcal{V} \llbracket z \rrbracket$ as well as $\forall g_{\mu_{1} \ldots \mu_{M+1}}^{(M)}\left(z_{1}, z_{2}\right) \in \mathcal{V} \llbracket z_{1}, z_{2} \rrbracket$. One proves the existence first when $f\left(z_{1}, z_{2}\right) \in \mathcal{V} \llbracket z_{1}, z_{2} \rrbracket$ by the change of variables $\left(z_{1}, z_{2}\right) \mapsto\left(z_{12}=z_{1}-z_{2}, z_{2}\right)$. In the general case: $f\left(z_{1}, z_{2}\right)=\left(z_{1}^{2} z_{2}^{2}\right)^{-N} \phi\left(z_{1}, z_{2}\right)$ for $N \gg 0$ and $\phi\left(z_{1}, z_{2}\right) \in$ $\mathcal{V} \llbracket z_{1}, z_{2} \rrbracket$. Then it is sufficient to prove that there exists the decomposition

$$
f\left(z_{1}, z_{2}\right)=f\left(z_{2}, z_{2}\right)+\sum_{\mu=1}^{D} z_{12}^{\mu} g_{\mu}^{(1)}\left(z_{1}, z_{2}\right),
$$

where $g_{\mu}^{(1)}\left(z_{1}, z_{2}\right) \in \mathcal{V} \llbracket z_{1}, z_{2} \rrbracket z_{1}^{2} z_{2}^{2}$. The existence of such a decomposition follows, on the other hand, from

$$
\begin{gathered}
f\left(z_{1}, z_{2}\right)-f\left(z_{2}, z_{2}\right)= \\
=\left(z_{1}^{2} z_{2}^{2}\right)^{-N}\left(\phi\left(z_{1}, z_{2}\right)-\phi\left(z_{2}, z_{2}\right)\right)+\phi\left(z_{2}, z_{2}\right)\left(\left(z_{1}^{2} z_{2}^{2}\right)^{-N}-\left(z_{2}^{2}\right)^{-2 N}\right), \\
\left(z_{1}^{2} z_{2}^{2}\right)^{-N}-\left(z_{2}^{2}\right)^{-2 N}= \\
=\left(z_{1}^{2}\right)^{-N}\left(z_{2}^{2}\right)^{-2 N}\left(\sum_{\mu=1}^{D} z_{12}^{\mu}\left(z_{1}^{\mu}+z_{2}^{\mu}\right)\right)\left(\sum_{k=0}^{N-1}\left(z_{1}^{2}\right)^{k}\left(z_{2}^{2}\right)^{N-k-1}\right) . \quad \square
\end{gathered}
$$

Lemma 2.3. For every $M \in \mathbb{N}$ and $P(z) \in \mathbb{C}[z]$ there exist $N \in \mathbb{N}$ and $Q(z, w) \in$ $\mathbb{C}[z, w]$ such that:

$$
\left(z^{2}\right)^{N} P\left(\partial_{z}\right)=Q\left(z, \partial_{z}\right)\left(z^{2}\right)^{M}
$$

where the equation is understood as an operator equality and $Q\left(z, \partial_{z}\right)$ stands for the polynomial $Q(z, w)$ with each monomial $z^{\mu_{1}} \ldots z^{\mu_{k}} w^{\nu_{1}} \ldots w^{\nu_{l}}$ replaced by $z^{\mu_{1}} \ldots z^{\mu_{k}}$ $\partial_{z^{\nu_{1}}} \ldots \partial_{z^{\nu_{l}}}$. 
Proof. Apply induction in $\operatorname{deg} P$. If $\operatorname{deg} P=0$ then $N=M$ and $Q=P$. If $\operatorname{deg} P>$ 0 then $P(z)=\sum_{\mu=1}^{D} z^{\mu} P_{\mu}(z)+P_{0}(z)$ where $\operatorname{deg} P_{\mu}(z)<\operatorname{deg} P(z)$ for $\mu=$ $0, \ldots, D$. By induction: for every $\mu=0, \ldots, D$ there exist $N_{\mu} \in \mathbb{N}$ and $Q_{\mu}^{\prime}(z, w) \in$ $\mathbb{C}[z, w]$ such that $\left(z^{2}\right)^{N_{\mu}} P\left(\partial_{z}\right)=Q_{\mu}^{\prime}\left(z, \partial_{z}\right)\left(z^{2}\right)^{M+1}$. Then let $N=\max \left\{N_{\mu}\right.$ : $\mu=0, \ldots, D\}$ so that $\left(z^{2}\right)^{N} P\left(\partial_{z}\right)=Q_{\mu}^{\prime \prime}\left(z, \partial_{z}\right)\left(z^{2}\right)^{M+1}$. Thus:

$$
\begin{gathered}
\left(z^{2}\right)^{N} P\left(\partial_{z}\right)= \\
=\left(\sum_{\mu=1}\left(Q_{\mu}^{\prime \prime}\left(z, \partial_{z}\right) \partial_{z^{\mu}} z^{2}-2(M+1) Q_{\mu}^{\prime \prime}\left(z, \partial_{z}\right) z^{\mu}\right)+Q_{0}^{\prime \prime}\left(z, \partial_{z}\right) z^{2}\right)\left(z^{2}\right)^{M} .
\end{gathered}
$$

Proof of Theorem 2.1 The first part of the theorem follows from Lemma 2.2 For the last statement we have to prove that:

$$
\left(z_{12}^{2}\right)^{N}\left[c\left(z_{1}\right), a\left(z_{2}\right)_{\{n, m, \sigma\}} b\left(z_{2}\right)\right] v=0 \text { for } N \gg 0 \text { and } v \in \mathcal{V}
$$

Because of the equality

$$
\left(a(z)_{\{n, m, \sigma\}} b(z)\right)^{(\varepsilon)}=\sum_{\varepsilon_{1}=0,1 \bmod 2} a^{\left(\varepsilon+\varepsilon_{1}\right)}(z)_{\{n, m, \sigma\}} b^{\left(\varepsilon_{1}\right)}(z)
$$

(using the notations of Definition 2.3) it is sufficient to consider the case when the fields $a(z), b(z)$ and $c(z)$ have fixed parities $p_{a}, p_{b}, p_{c} \in \mathbb{Z}_{2}$, respectively. Then we have

$$
\begin{aligned}
& \left(z_{12}^{2}\right)^{N+M} c\left(z_{1}\right) a\left(z_{2}\right)_{\{n, m, \sigma\}} b\left(z_{2}\right) v= \\
= & \left.\left(z_{12}^{2}\right)^{N}\left(z_{13}^{2}\right)^{M} P_{\{n+M, m, \sigma\}}\left(\partial_{z_{2}}\right)\left(z_{23}^{2}\right)^{M} c\left(z_{1}\right) a\left(z_{2}\right) b\left(z_{3}\right) v\right|_{z_{3}=z_{2}}= \\
= & \left.Q\left(z_{12}, \partial_{z_{2}}\right)\left(z_{12}^{2}\right)^{M}\left(z_{13}^{2}\right)^{M}\left(z_{23}^{2}\right)^{M} c\left(z_{1}\right) a\left(z_{2}\right) b\left(z_{3}\right) v\right|_{z_{3}=z_{2}}= \\
= & \left.(-1)^{p_{a} p_{c}+p_{b} p_{c}} Q\left(z_{12}, \partial_{z_{2}}\right)\left(z_{12}^{2}\right)^{M}\left(z_{13}^{2}\right)^{M}\left(z_{23}^{2}\right)^{M} a\left(z_{2}\right) b\left(z_{3}\right) c\left(z_{1}\right) v\right|_{z_{3}=z_{2}}= \\
= & (-1)^{p_{a} p_{c}+p_{b} p_{c}}\left(z_{12}^{2}\right)^{N}\left(z_{13}^{2}\right)^{M} \times \\
& \times\left. P_{\{n+M, m, \sigma\}}\left(\partial_{z_{2}}\right)\left(z_{23}^{2}\right)^{M} a\left(z_{2}\right) b\left(z_{3}\right) c\left(z_{1}\right) v\right|_{z_{3}=z_{2}}= \\
= & (-1)^{\left(p_{a}+p_{b}\right) p_{c}}\left(z_{12}^{2}\right)^{N+M} a\left(z_{2}\right)_{\{n, m, \sigma\}} b\left(z_{2}\right) c\left(z_{1}\right) v,
\end{aligned}
$$

for sufficiently large $M$ and $N$, independent on $v \in \mathcal{V}$, in accord with Eq. (2.5) and Lemma 2.3

The $\{0,0,1\}$-product is the natural candidate for the notion of normal product in vertex algebras which generalizes the corresponding one from the chiral CFT:

$$
: Y(a, z) Y(b, z)::=Y(a, z)_{\{0,0,1\}} Y(b, z) .
$$

As a consequence of Eqs. (2.5) and (1.12) it could be expressed as:

$$
: Y(a, z) Y(b, z): v=\left.K_{N}\left(\partial_{z_{1}}^{2}\right)^{N}\left[\left(z_{12}^{2}\right)^{N} Y\left(a, z_{1}\right) Y\left(b, z_{2}\right)\right] v\right|_{z_{1}=z_{2}=z}
$$

for $N \gg 0$ and every $v \in \mathcal{V}$. 


\section{Consequences of the Existence of a Vacuum and of Translation Invariance}

There is a vertex algebra analog of (the corollary of) the Reeh-Schlider theorem - the separating property of the vacuum [4].

Theorem 3.1. Let $\mathcal{V}$ be a vertex algebra and $u(z)$ be a field on $\mathcal{V}$ which is mutually local with respect to all fields $Y(a, z), a \in \mathcal{V}$. Then if $u(z) \widehat{1}=0$ it follows that $u(z)=0$.

Proof. Because of locality we have for every $a \in \mathcal{V}$ and $N_{a} \gg 0$ :

$$
\left(z_{12}^{2}\right)^{N_{a}} u\left(z_{1}\right) Y\left(a, z_{2}\right) \hat{1}=\left(z_{12}^{2}\right)^{N_{a}} Y\left(a, z_{2}\right) u\left(z_{1}\right) \hat{1},
$$

thus obtaining $\left(z_{12}^{2}\right)^{N_{a}} u\left(z_{1}\right) Y\left(a, z_{2}\right) \hat{1}=0$. Then we can set $z_{2}=0$ and divide by $\left(z_{12}^{2}\right)^{N_{a}}=\left(z_{1}^{2}\right)^{N_{a}}$ because it multiplies an element of $\mathcal{V} \llbracket z_{1} \rrbracket_{z_{1}^{2}}$ (in the $\left(\mathbb{C} \llbracket z \rrbracket_{z^{2}}\right)$ module $\mathcal{V} \llbracket z \rrbracket_{z^{2}}$ there are no zero divisors). Thus we obtain that $u(z) a=0$ for every $a \in \mathcal{V}$.

The following proposition shows that the system of fields $\{Y(a, z): a \in \mathcal{V}\}$ is a maximal system of translation invariant local fields.

Proposition 3.2. Let $\mathcal{V}$ be a vertex algebra and $u(z)$ be a field on $\mathcal{V}$ which is mutually local with respect to all fields $Y(a, z), a \in \mathcal{V}$. Then the following conditions are equivalent:

(a) $\left[T_{\mu}, u(z)\right]=\partial_{z^{\mu}} u(z)$ for $\mu=1, \ldots, D$ and $u(z) \hat{1} \in \mathcal{V} \llbracket z \rrbracket$, as for $z=0$, $\left.u(z) \hat{1}\right|_{z=0}=c$;

(b) $u(z) \widehat{1}=\exp (T \cdot z) c$, where $T \cdot z \equiv \sum_{\mu=1}^{D} T_{\mu} z^{\mu}$ and $\exp (T \cdot z)=$ $\sum_{n=0}^{\infty} \frac{1}{n !}(T \cdot z)^{n} \in($ End $\mathcal{V}) \llbracket z \rrbracket ;$

(c) $u(z)=Y(c, z)$.

Proof. $(a) \Rightarrow(b)$. The equality $u(z) \hat{1}=\exp (T \cdot z) c$ appears as the unique solution of the equations $\partial_{z^{\mu}}(u(z) \hat{1})=T_{\mu}(u(z) \hat{1})$ for $\mu=1, \ldots, D$ with initial condition $\left.u(z) \hat{1}\right|_{z=0}=c$. Indeed, if

$$
u(z) \widehat{1}=\sum_{n=0}^{\infty} \sum_{\mu_{1}, \ldots, \mu_{n}=1}^{D} c_{\mu_{1} \ldots \mu_{n}}^{(n)} z^{\mu_{1}} \ldots z^{\mu_{n}}, \quad c_{\mu_{1} \ldots \mu_{n}}^{(n)} \in \mathcal{V},
$$

then $c^{(0)}=c$ and $c_{\mu_{1} \ldots \mu_{n}}^{(n)}=\frac{1}{n} T_{\mu_{1}} c_{\mu_{2} \ldots \mu_{n}}^{(n-1)}=\cdots=\frac{1}{n !} T_{\mu_{1}} \ldots T_{\mu_{n}} c$ for $n>1$.

$(b) \Rightarrow(c)$. By Definition 2.1 $(c)$ and $(d)$, and the implication $(a) \Rightarrow(b)$ above we have $Y(c, z) \hat{1}=\exp (T \cdot z) c$. Then $(u(z)-Y(c, z)) \hat{1}=0$ and by Theorem 3.1 we conclude that $u(z)=Y(c, z)$.

$(c) \Rightarrow(a)$. This is a part of Definition 2.1 (conditions $(c)$ and $(d)$ ).

Corollary 3.3. Let $\mathcal{V}$ be a vertex algebra. Then for all $a \in \mathcal{V}$ and $\mu=1, \ldots, D$ :

$$
Y\left(T_{\mu} a, z\right)=\partial_{z^{\mu}} Y(a, z) \equiv\left[T_{\mu}, Y(a, z)\right] .
$$


Proof. Eq. 3.1) follows from the equality and Proposition 3.2

$$
\left.Y\left(T_{\mu} a, z\right) \hat{1}\right|_{z=0}=T_{\mu} a=\left.\left[T_{\mu}, Y(a, z)\right] \hat{1}\right|_{z=0}
$$

Proposition 3.4. Let $\mathcal{V}$ be a vertex algebra. Then for all $a, b \in \mathcal{V}$ and $n \in \mathbb{Z}, m=$ $0,1, \ldots, \sigma=1, \ldots, \mathfrak{h}_{m}$ :

$$
Y(a, z)_{\{n, m, \sigma\}} Y(b, z)=Y\left(a_{\{n, m, \sigma\}} b, z\right),
$$

and for $n \geqslant 0$ :

$$
Y(a, z)_{\{n, m, \sigma\}} Y(b, z)=\left(P_{\{n, m, \sigma\}}\left(\partial_{z}\right) Y(a, z)\right)_{\{0,0,1\}} Y(b, z) .
$$

Proof. To prove Eq. (3.2) we will basically use Eq. 2.5. First we have for $N \gg 0$ and all $v \in \mathcal{V}, \mu=1, \ldots, D$ :

$$
\begin{aligned}
& {\left[T_{\mu}, Y(a, z)_{\{n, m, \sigma\}} Y(b, z)\right] v=} \\
= & \left.P_{\{n+N, m, \sigma\}}\left(\partial_{z_{1}}\right)\left(z_{12}^{2}\right)^{N}\left[T_{\mu}, Y\left(a, z_{1}\right) Y\left(b, z_{2}\right)\right] v\right|_{z_{1}=z_{2}=z}= \\
= & P_{\{n+N, m, \sigma\}}\left(\partial_{z_{1}}\right)\left(z_{12}^{2}\right)^{N} \times \\
& \times\left.\left(\partial_{z_{1}^{\mu}} Y\left(a, z_{1}\right) Y\left(b, z_{2}\right)+Y\left(a, z_{1}\right) \partial_{z_{2}^{\mu}} Y\left(b, z_{2}\right)\right) v\right|_{z_{1}=z_{2}=z}= \\
= & \left.P_{\{n+N, m, \sigma\}}\left(\partial_{z_{1}}\right)\left(\partial_{z_{1}^{\mu}}+\partial_{z_{2}^{\mu}}\right)\left(z_{12}^{2}\right)^{N} Y\left(a, z_{1}\right) Y\left(b, z_{2}\right) v\right|_{z_{1}=z_{2}=z}= \\
= & \partial_{z^{\mu}}\left[\left.P_{\{n+N, m, \sigma\}}\left(\partial_{z_{1}}\right)\left(z_{12}^{2}\right)^{N} Y\left(a, z_{1}\right) Y\left(b, z_{2}\right) v\right|_{z_{1}=z_{2}=z}\right]= \\
= & \partial_{z^{\mu}}\left(Y(a, z)_{\{n, m, \sigma\}} Y(b, z) v\right) .
\end{aligned}
$$

On the other hand, for $N \gg 0$ :

$$
\begin{gathered}
\left.Y(a, z)_{\{n, m, \sigma\}} Y(b, z) \hat{1}\right|_{z=0}= \\
=\left[\left.P_{\{n+N, m, \sigma\}}\left(\partial_{z_{1}}\right)\left(z_{12}^{2}\right)^{N} Y\left(a, z_{1}\right) Y\left(b, z_{2}\right) \hat{1}\right|_{z_{1}=z_{2}=z}\right]_{z=0} .
\end{gathered}
$$

But $P_{\{n+N, m, \sigma\}}\left(\partial_{z_{1}}\right)\left(z_{12}^{2}\right)^{N} Y\left(a, z_{1}\right) Y\left(b, z_{2}\right) \hat{1} \in \mathcal{V} \llbracket z_{1}, z_{2} \rrbracket$, so that the consecutive restrictions $z_{1}=z_{2}=z$ and $z=0$ are equivalent to the restrictions: first $z_{2}=0$ and then $z_{1}=0$. In such a way we obtain that

$$
\left.Y(a, z)_{\{n, m, \sigma\}} Y(b, z) \hat{1}\right|_{z=0}=a_{\{n, m, \sigma\}} b .
$$

Combining these two results we conclude by Proposition 3.2 that Eq. (3.2) holds.

The proof of (3.3) uses Eqs. (3.2), 2.2) and some additional properties of the polynomials $P_{\{n, m, \sigma\}}(z)$. We will not prove (3.3) since we will not use it further. 
Corollary 3.5. Let $u(z)=\sum_{n \in \mathbb{Z}} \sum_{m=0}^{\infty} \sum_{\sigma=1}^{\mathfrak{h}_{m}} u_{\{n, m, \sigma\}}\left(z^{2}\right)^{n} h_{\sigma}^{(m)}(z)$ and $v(z)$ be two mutually local fields on a superspace $\mathcal{V}$ and $\hat{1} \in \mathcal{V}_{0}$ be such that $u(z) \hat{1}$ and $v(z) \widehat{1}$ belong to $\in \mathcal{V} \llbracket z \rrbracket$. Then

$$
\left.u(z)_{\{n, m, \sigma\}} v(z) \hat{1}\right|_{z=0}=u_{\{n, m, \sigma\}}\left(\left.v(z) \hat{1}\right|_{z=0}\right) .
$$

Proof. This can be derived as in the proof of Proposition 3.4 (the derivation of (3.5).

\section{Existence Theorem. Analytic Continuations}

The next theorem allows one to construct a vertex algebra from a system of mutually local and "translation covariant" fields which give rise to the entire space by acting on the vacuum.

Theorem 4.1. ("Existence Theorem”.)

Let $u^{\alpha}(z)=\sum_{n \in \mathbb{Z}} \sum_{m=0}^{\infty} \sum_{\sigma=1}^{\mathfrak{h}_{m}} u_{\{n, m, \sigma\}}^{\alpha}\left(z^{2}\right)^{n} h_{\sigma}^{(m)}(z)$ for $\alpha \in \mathcal{A}$ be a system of mutually local fields on a superspace $\mathcal{V}$. Let $\widehat{1} \in \mathcal{V}_{0}$ and $T_{\mu} \in(\text { End } \mathcal{V})_{0}$ be such that $T_{\mu} \widehat{1}=0$ for $\mu=1, \ldots, D$ and:

(a) $\left[T_{\mu}, u^{\alpha}(z)\right]=\partial_{z^{\mu}} u^{\alpha}(z)$ and $u^{\alpha}(z) \hat{1} \in \mathcal{V} \llbracket z \rrbracket$ for all $\alpha \in \mathcal{A}, \mu=1, \ldots, D$;

(b) the set of all elements $u_{\left\{n_{1}, m_{1}, \sigma_{1}\right\}}^{\alpha_{1}} \ldots u_{\left\{n_{N}, m_{N}, \sigma_{N}\right\}}^{\alpha_{N}} \widehat{1}$ for $N=0,1, \ldots$, $\alpha_{k} \in \mathcal{A}, n_{k} \in \mathbb{Z}, n_{N} \geqslant 0, m_{k}=0,1, \ldots, \sigma_{k}=1, \ldots, \mathfrak{h}_{m_{k}}(k=$ $1, \ldots, N)$, spans the space $\mathcal{V}$.

Then there exists a unique structure of a vertex algebra with vacuum $\widehat{1}$ and translation endomorphisms $T_{\mu}$ on $\mathcal{V}$ such that

$$
Y\left(u^{\alpha}, z\right)=u^{\alpha}(z) \quad \text { for } \quad u^{\alpha}:=\left.u^{\alpha}(z) \hat{1}\right|_{z=0}, \quad \alpha \in \mathcal{A}
$$

The operators $Y(a, z)$ are determined for the vectors of the set in the above condition (b) by:

$$
\begin{gathered}
Y\left(u_{\left\{n_{1}, m_{1}, \sigma_{1}\right\}}^{\alpha_{1}} \ldots u_{\left\{n_{N}, m_{N}, \sigma_{N}\right\}}^{\alpha_{N}} \hat{1}, z\right)= \\
=u^{\alpha_{1}}(z)_{\left\{n_{1}, m_{1}, \sigma_{1}\right\}}\left(\ldots u ^ { \alpha _ { N - 1 } } ( z ) _ { \{ n _ { N - 1 } , m _ { N - 1 } , \sigma _ { N - 1 } \} } \left(P_{\left\{n_{N}, m_{N}, \sigma_{N}\right\}}\left(\partial_{z}\right) \times\right.\right. \\
\left.\left.\times u^{\alpha_{N}}(z)\right) \ldots\right) .
\end{gathered}
$$

Proof. Set $Y(\widehat{1}, z)=\mathbb{I}$ and take Eq. (4.2) as a definition for the operators $Y(a, z)$ restricting to a subsystem of the set displayed in condition $(b)$ which contains $\widehat{1}$ and forms a basis of $\mathcal{V}$. By Theorem 2.1 we obtain a system of mutually local fields. The conditions $(c)$ and $(d)$ of Definition 2.1 can be proven by induction in $N$ for the fields 4.2 following the argument of the first part of the proof of Proposition 3.4 (the computations of (3.4) and (3.5). The uniqueness follows from Proposition 3.2 
Now we will find an analogue of the analytic continuation of products of Wightman fields acting on the vacuum.

Let $R$ be a ring and $V$ be an $R$-module. Then $V \llbracket z \rrbracket_{z^{2}}$ is an $\left(R \llbracket z \rrbracket_{z^{2}}\right)$-module with derivations $\partial_{z^{\mu}}$ for $\mu=1, \ldots D$. Moreover, if the $R$-module $V$ has no zero divisors then this is also true for the $\left(R \llbracket z \rrbracket z^{2}\right)$-module $V \llbracket z \rrbracket z^{2}$.

From this simple fact it follows by induction that

$$
V \llbracket z_{1} \rrbracket z_{1}^{2} \ldots \llbracket z_{n} \rrbracket_{z_{n}^{2}}:=\left(V \llbracket z_{1} \rrbracket z_{1}^{2} \ldots\right) \llbracket z_{n} \rrbracket z_{n}^{2}
$$

is a $\left(\mathbb{C} \llbracket z_{1} \rrbracket_{z_{1}^{2}} \ldots \llbracket z_{n} \rrbracket_{z_{n}^{2}}\right)$-module with derivations $\partial_{z_{k}^{\mu}}(k=1, \ldots, n, \mu=1, \ldots$, $D)$, which has no zero divisors. Note that

$$
V \llbracket z_{1}, \ldots, z_{n} \rrbracket_{z_{1}^{2} \ldots z_{n}^{2}} \varsubsetneqq V \llbracket z_{1} \rrbracket_{z_{1}^{2}} \ldots \llbracket z_{n} \rrbracket z_{n}^{2} \varsubsetneqq V \llbracket z_{1}, 1 / z_{1}^{2} ; \ldots ; z_{n}, 1 / z_{n}^{2} \rrbracket .
$$

It follows from the definition of vertex algebra (Def.2.1) that in a vertex algebra $\mathcal{V}$, for all $a_{1}, \ldots, a_{n}, b \in \mathcal{V}$ :

$$
Y\left(a_{1}, z_{1}\right) \ldots Y\left(a_{n}, z_{n}\right) b \in \mathcal{V} \llbracket z_{1} \rrbracket z_{1}^{2} \ldots \llbracket z_{n} \rrbracket_{z_{n}^{2}}
$$

Let us introduce the following multiplicative systems in $\mathbb{C}\left[z_{1}, \ldots, z_{n}\right]$ :

$$
\begin{gathered}
L_{n}:=\left\{\prod_{k=1}^{N}\left(\sum_{l=1}^{n} \lambda_{k, l} z_{l}\right)^{2}: N \in \mathbb{N},\left(\lambda_{k, 1}, \ldots, \lambda_{k, n}\right) \in \mathbb{C}^{n} \backslash\{0\}\right. \\
\text { for } k=1, \ldots, N\}, \\
R_{n}:=\left\{\left(\prod_{k=1}^{N} z_{k}^{2}\right)^{N}\left(\prod_{1 \leqslant l<m \leqslant n} z_{l m}^{2}\right)^{N}: N \in \mathbb{N}\right\} \\
\left.z_{l m}=z_{l}-z_{m} \in \mathbb{C}\left[z_{l}, z_{m}\right]\right) . \text { Clearly, } \\
R_{n} \varsubsetneqq L_{n} \text { and } V \llbracket z_{1}, \ldots, z_{n} \rrbracket_{R_{n}} \varsubsetneqq V \llbracket z_{1}, \ldots, z_{n} \rrbracket_{L_{n}}
\end{gathered}
$$

for every vector space $V$ as the localized modules in (4.8) have induced derivations $\partial_{z^{\mu}}$ $(\mu=1, \ldots, D)$.

For every linear automorphism $A: \mathbb{C}^{n} \rightarrow \mathbb{C}^{n}$ with matrix $\left(A_{k l}\right)$ and inverse matrix $\left({ }^{-1} k l\right)$, and a vector space $V$ we define an induced automorphism

$$
r(A): V \llbracket z_{1}, \ldots, z_{n} \rrbracket_{L_{n}} \longrightarrow V \llbracket z_{1}, \ldots, z_{n} \rrbracket_{L_{n}},
$$

the "linear change of variables $z_{k} \mapsto z_{k}^{\prime}=\sum_{l=1}^{n} A_{k l} z_{l}$ ", replacing $z_{k} \mapsto \sum_{l=1}^{n}{ }_{A}^{-1} z_{k l}$ $(k=1, \ldots, n)$ (note that the multiplicative system $L_{n}$ (4.6) is invariant under this replacement while $R_{n}$ is not). There is also a natural action of the symmetric group $\mathcal{S}_{n}$ on $V \llbracket z_{1}, \ldots, z_{n} \rrbracket_{L_{n}}$ and $V \llbracket z_{1}, \ldots, z_{n} \rrbracket_{R_{n}}$ induced by the permutation of variables 
$\left(z_{1}, \ldots, z_{n}\right)$, since the multiplicative systems $L_{n}$ and $R_{n}$ are invariant under this action.

Now we will introduce a homomorphism, commuting with the derivations $\partial_{z^{\mu}}$,

$$
\iota_{z_{1}, \ldots, z_{n}}: \mathbb{C} \llbracket z_{1}, \ldots, z_{n} \rrbracket_{L_{n}} \longrightarrow \mathbb{C} \llbracket z_{1} \rrbracket_{z_{1}^{2}} \ldots \llbracket z_{n} \rrbracket z_{n}^{2}
$$

that will be the expansion "in the domain $\left|z_{1}^{2}\right|>\cdots>\left|z_{n}^{2}\right|$ ". We first set

$$
\left.\iota_{z_{1}, \ldots, z_{n}}\right|_{\mathbb{C} \llbracket z_{1}, \ldots, z_{n} \rrbracket}=\mathbb{I}_{\mathbb{C} \llbracket z_{1}, \ldots, z_{n} \rrbracket} \cdot
$$

Next, consider for every $N \in \mathbb{Z}$ and constants $\left(\lambda_{1}, \ldots, \lambda_{n}\right) \in \mathbb{C}^{n}$, the Taylor expansions in the $D$-dimensional variables $z_{1}, \ldots, z_{n}$ :

$$
\begin{gathered}
\iota\left(1+2 \sum_{2 \leqslant k<l \leqslant n} \lambda_{1}^{2} \lambda_{k} \lambda_{l} z_{1}^{2} z_{k} \cdot z_{l}+\sum_{m=2}^{n}\left(2 \lambda_{1} \lambda_{m} z_{1} \cdot z_{m}+\lambda_{1}^{2} \lambda_{m}^{2} z_{1}^{2} z_{m}^{2}\right)\right)^{-N}= \\
=\sum_{k_{1}, \ldots, k_{n}=0}^{\infty} \lambda_{1}^{k_{1}} \ldots \lambda_{n}^{k_{n}} f_{k_{1} \ldots k_{n}}^{N}\left(z_{1}, \ldots, z_{n}\right) \in \mathbb{C}\left[z_{1}\right] \llbracket z_{2}, \ldots, z_{n} \rrbracket \subset \\
\subset \mathbb{C} \llbracket z_{1}, \ldots, z_{n} \rrbracket
\end{gathered}
$$

where $f_{k_{1} \ldots k_{n}}^{N}\left(z_{1}, \ldots, z_{n}\right)$ are separately homogeneous polynomials in $z_{1}, \ldots, z_{n}$ of degrees $k_{1}, \ldots, k_{n}$, respectively, and the coefficient (formal) series in $z_{1}$ for every monomial in $z_{2}, \ldots, z_{n}$ is actually a polynomial. (The last is true because the polynomials $f_{k_{1} \ldots k_{n}}^{N}\left(z_{1}, \ldots, z_{n}\right)$ are zero if $k_{1}>k_{2}+\cdots+k_{n}$.) Thus we can replace $z_{1}$ by $\frac{z_{1}}{z_{1}^{2}}$ in the formal series (4.12) and define for every $N \in \mathbb{Z}$ and constants $\left(\lambda_{1}, \ldots, \lambda_{n}\right) \in \mathbb{C}^{n}, \lambda_{1} \neq 0$ :

$$
\begin{gathered}
\iota_{z_{1}, \ldots, z_{n}}\left(\left(\sum_{k=1}^{n} \lambda_{k} z_{l}\right)^{2}\right)^{-N}:=\left(\lambda_{1}^{2} z_{1}^{2}\right)^{-N}\left[\iota \left(1+2 \sum_{2 \leqslant k<l \leqslant n} \lambda_{1}^{-2} \lambda_{k} \lambda_{l} z_{1}^{2} z_{k} \cdot z_{l}+\right.\right. \\
\left.\left.+\sum_{m=2}^{n}\left(2 \lambda_{1}^{-1} \lambda_{m} z_{1} \cdot z_{m}+\lambda_{1}^{-2} \lambda_{m}^{2} z_{1}^{2} z_{m}^{2}\right)\right)\left.^{-N}\right|_{\left.z_{1} \mapsto \frac{z_{1}}{z_{1}^{2}}\right]}\right] \\
\in \mathbb{C}\left[z_{1}, 1 / z_{1}^{2}\right] \llbracket z_{2}, \ldots, z_{n} \rrbracket \subset \mathbb{C} \llbracket z_{1} \rrbracket z_{1}^{2} \ldots \llbracket z_{n} \rrbracket z_{n}^{2}
\end{gathered}
$$

For general constants $\left(\lambda_{1}, \ldots, \lambda_{n}\right) \in \mathbb{C}^{n} \backslash\{0\}$ we set

$$
\iota_{z_{1}, \ldots, z_{n}}\left(\left(\sum_{k=1}^{n} \lambda_{k} z_{l}\right)^{2}\right)^{-N}:=\iota_{z_{m}, \ldots, z_{n}}\left(\left(\sum_{k=m}^{n} \lambda_{k} z_{l}\right)^{2}\right)^{-N}
$$

$\left(\in \mathbb{C} \llbracket z_{1} \rrbracket z_{1}^{2} \ldots \llbracket z_{n} \rrbracket z_{n}^{2}\right)$ if $\lambda_{1}=\cdots=\lambda_{m-1}=0, \lambda_{m} \neq 0$. Since the Taylor expansions 4.12) have a multiplicative property, then

$$
\begin{gathered}
\iota_{z_{1}, \ldots, z_{n}}\left(\left(\sum_{k=1}^{n} \lambda_{k} z_{k}\right)^{2}\right)^{-N_{1}} \iota_{z_{1}, \ldots, z_{n}}\left(\left(\sum_{k=1}^{n} \lambda_{k} z_{k}\right)^{2}\right)^{-N_{2}}= \\
=\iota_{z_{1}, \ldots, z_{n}}\left(\left(\sum_{k=1}^{n} \lambda_{k} z_{k}\right)^{2}\right)^{-N_{1}-N_{2}}
\end{gathered}
$$


for $N_{1}, N_{2} \in \mathbb{Z}$. Finally, the homomorphism $\iota_{z_{1}, \ldots, z_{n}}$ (4.10) is uniquely determined by Eqs. 4.11-4.15.

Remark 4.1. The operation $\iota_{z_{1}, \ldots, z_{n}}$ applied to a rational function $R\left(z_{1}, \ldots, z_{n}\right)$, regular for $z_{2}=0, \ldots, z_{n}=0$, should give the Taylor expansion of $R$ in $z_{2}, \ldots, z_{n}$ around $(0, \ldots, 0)$. Its coefficients are rational functions in $z_{1}$. In the case of the left hand side of (4.13) these coefficients are polynomials in $z_{1}$ and ${ }^{1} / z_{1}^{2}$, which follows by induction in the total order of $z_{2}, \ldots, z_{n}$. (The author thanks A. Retakh for his interest in this work and for asking a question answered in this Remark.)

Note that $\iota_{z_{1}}, \ldots, z_{n}$ is a $\mathbb{C}\left[z_{1}, 1 / z_{1}^{2} ; \ldots ; z_{n}, 1{ }_{z_{n}^{2}}\right]$-linear map and commutes with the derivations $\partial_{z_{k}^{\mu}}(k=1, \ldots, n, \mu=1, \ldots, D)$. We can also define a $\mathbb{C}\left[z_{1}, 1 / z_{1}^{2} ; \ldots\right.$; $\left.z_{n}, 1 / z_{n}^{2}\right]$-linear map

\[ \iota_{z_{1}, \ldots, z_{n}}: V \llbracket z_{1}, \ldots, z_{n} \rrbracket_{L_{n}} \rightarrow V \llbracket z_{1} \rrbracket_{z_{1}^{2}} \ldots \llbracket z_{n} \rrbracket_{z_{n}^{2}}, \]
so that $\left.\iota_{z_{1}, \ldots, z_{n}}\right|_{V \llbracket z_{1}, \ldots, z_{n} \rrbracket}=\mathbb{I}_{V \llbracket z_{1}, \ldots, z_{n} \rrbracket}$ and $\iota_{z_{1}, \ldots, z_{n}}(f u)=\iota_{z_{1}, \ldots, z_{n}}(f)$ $\iota_{z_{1}, \ldots, z_{n}}(u)$ for $f \in \mathbb{C} \llbracket z_{1}, \ldots, z_{n} \rrbracket_{L_{n}}$ and $u \in V \llbracket z_{1}, \ldots, z_{n} \rrbracket_{L_{n}}$.

The map $\iota_{z_{1}, \ldots, z_{n}}$ has zero kernel in $V \llbracket z_{1}, \ldots, z_{n} \rrbracket_{L_{n}}$. Indeed, if $\iota_{z_{1}, \ldots, z_{n}} u=0$ for some $u \in V \llbracket z_{1}, \ldots, z_{n} \rrbracket_{L_{n}}$ then $u=f^{-1} v$, where $f:=\prod_{k=1}^{N}\left(\sum_{l=1}^{n} \lambda_{k, l} z_{l}\right)^{2}, v \in$ $V \llbracket z_{1}, \ldots, z_{n} \rrbracket$. But $\iota_{z_{1}}, \ldots, z_{n} u=\iota_{z_{1}}, \ldots, z_{n}\left(f^{-1}\right) \iota_{z_{1}, \ldots, z_{n}} v$ and $\iota_{z_{1}}, \ldots, z_{n} v \equiv v \neq 0$, $\iota_{z_{1}, \ldots, z_{n}}\left(f^{-1}\right) \neq 0$ (since $\iota_{z_{1}}, \ldots, z_{n}\left(f^{-1}\right)$ is the inverse of $f$ ), which contradicts the fact that in $V \llbracket z_{1} \rrbracket z_{1}^{2} \ldots \llbracket z_{n} \rrbracket_{z_{n}^{2}}$ there are no zero divisors.

Proposition 4.2. In any vertex algebra $\mathcal{V}$ and $a_{1}, \ldots, a_{n}, b \in \mathcal{V}$ one has

$$
\begin{gathered}
Y\left(a_{1}, z_{1}\right) \ldots Y\left(a_{n}, z_{n}\right) b \in \iota_{z_{1}, \ldots, z_{n}}\left(\mathcal{V} \llbracket z_{1}, \ldots, z_{n} \rrbracket_{R_{n}}\right) \subset \\
\subset \mathcal{V} \llbracket z_{1} \rrbracket_{z_{1}^{2}} \ldots \llbracket z_{n} \rrbracket_{z_{n}^{2}}
\end{gathered}
$$

(see Eq. (4.7)). Moreover, the inverse image

$$
\begin{aligned}
\mathcal{Y}_{n}\left(a_{1}, z_{1} ; \ldots ; a_{n}, z_{n} ; b\right) & :=\iota_{z_{1}, \ldots, z_{n}}^{-1}\left(Y\left(a_{1}, z_{1}\right) \ldots Y\left(a_{n}, z_{n}\right) b\right) \in \\
& \in \mathcal{V} \llbracket z_{1}, \ldots, z_{n} \rrbracket_{R_{n}}
\end{aligned}
$$

is $\mathbb{Z}_{2}$-symmetric in the sense that if $a_{1}, \ldots a_{n}$ have fixed parities $p_{1}, \ldots p_{n}$ (resp.) then for any permutation $\sigma \in \mathcal{S}_{n}$ :

$\mathcal{Y}_{n}\left(a_{\sigma(1)}, z_{\sigma(1)} ; \ldots ; a_{\sigma(n)}, z_{\sigma(n)} ; b\right)=(-1)^{\varepsilon(\sigma)} \mathcal{Y}_{n}\left(a_{1}, z_{1} ; \ldots ; a_{n}, z_{n} ; b\right)$,

where $\varepsilon(\sigma):=\sum_{(i j)} p_{a_{i}} p_{a_{j}}$ mod 2 (the sum is taken over all transpositions $(i j)$ in some representation $\sigma=\prod(i j)$ ). 
Proof. Locality (Definition 2.1 (b)) implies that

$$
\rho_{n}^{N} Y\left(a_{1}, z_{1}\right) \ldots Y\left(a_{n}, z_{n}\right) b \in \mathcal{V} \llbracket z_{1}, \ldots, z_{n} \rrbracket \quad \text { for } \quad N \gg 0,
$$

where $\rho_{n}:=\left(\prod_{k} z_{k}^{2}\right)\left(\prod_{l<m} z_{l m}^{2}\right)$. On the other hand,

$$
Y\left(a_{1}, z_{1}\right) \ldots Y\left(a_{n}, z_{n}\right) b \in \mathcal{V} \llbracket z_{1} \rrbracket z_{1}^{2} \ldots \llbracket z_{n} \rrbracket_{z_{n}^{2}},
$$

because of Definition 2.1 (a). Then (4.16) follows from the fact that $\iota_{z_{1}}, \ldots, z_{n} \rho_{n}^{-N}$ is an inverse element of $\rho_{n}^{N}$ in $\mathbb{C} \llbracket z_{1} \rrbracket z_{1}^{2} \ldots \llbracket z_{n} \rrbracket_{z_{n}^{2}}$. To prove Eq. (4.18 we note that for $N \gg 0: \rho_{n}^{N} \mathcal{Y}_{n}\left(a_{1}, z_{1} ; \ldots ; a_{n}, z_{n} ; b\right)$ is $\mathbb{Z}_{2}$-symmetric, while $\rho_{n}^{N}$ is symmetric in $z_{1}, \ldots, z_{n}$.

Theorem 4.3. In any vertex algebra $\mathcal{V}$ and $a, b, c \in \mathcal{V}$ it follows that

$$
Y(Y(a, z-w) b, w) c=\iota_{w, z-w}\left(r_{z, w}^{w, z-w}\left(\iota_{z, w}^{-1}(Y(a, z) Y(b, w) c)\right)\right),
$$

where $Y(Y(a, z-w) b, w) c$ is viewed as a series belonging to $\mathcal{V} \llbracket w \rrbracket_{w^{2}} \llbracket z-w \rrbracket_{(z-w)^{2}}$, $\iota_{z, w}^{-1}$ is the inverse of $\iota_{z, w}$ on its image and $r_{z, w}^{w, z-w}: \mathcal{V} \llbracket z, w \rrbracket_{L_{n}} \rightarrow \mathcal{V} \llbracket w, z-w \rrbracket_{L_{n}}$ is the map of type (4.9) induced by the change of variables $(z, w) \mapsto(w, z-w)$.

Proof. The theorem follows from Theorem 2.1 (Eq. 2.4) and Eq. 3.2. More precisely, we obtain the following equalities in $\mathcal{V} \llbracket z, w \rrbracket \cong \mathcal{V} \llbracket w, z-w \rrbracket$ for $N \gg 0$ :

$$
\begin{gathered}
\left(z^{2} w^{2}(z-w)^{2}\right)^{N} Y(a, z) Y(b, w) c= \\
=\left(z^{2} w^{2}\right)^{N} \sum_{n, m=0}^{\infty} \sum_{\sigma=1}^{\mathfrak{h}_{m}} Y(a, w)_{\{n-N, m, \sigma\}} Y(b, w) c\left((z-w)^{2}\right)^{n} h_{\sigma}^{(m)}(z-w)= \\
=\left(z^{2} w^{2}\right)^{N} \sum_{n, m=0}^{\infty} \sum_{\sigma=1}^{\mathfrak{h}_{m}} Y\left(a_{\{n-N, m, \sigma\}} b, w\right) c\left((z-w)^{2}\right)^{n} h_{\sigma}^{(m)}(z-w)= \\
=\left.\left(z_{1}^{2} z_{2}^{2}\left(z_{1}+z_{2}\right)^{2}\right)^{N} Y\left(Y\left(a, z_{1}\right) b, z_{2}\right) c\right|_{z_{1}=z-w, z_{2}=w} ;
\end{gathered}
$$

then the prefactors can be cancelled after applying to both sides the corresponding $\iota^{-1}$ operators.

\section{Free Field Examples. Lie Superalgebras of Formal Distributions}

Let us consider a central extension of the free commutative Lie superalgebra

$$
\operatorname{Span}_{\mathbb{C}}\left\{u_{\{n, m, \sigma\}}^{\alpha}: \alpha \in A, n \in \mathbb{Z}, m \in N \cup\{0\}, \sigma=1, \ldots, \mathfrak{h}_{m}\right\},
$$

where $A$ is some index set and all generators $u_{\{n, m, \sigma\}}^{\alpha}$ have parities $p_{\alpha}$ which do not depend on $n, m$ and $\sigma$. The commutation relations are presented by the following generating functions:

$$
\begin{aligned}
{\left[u^{\alpha}(z), u^{\beta}(w)\right] } & =\left(\iota_{z, w} \frac{Q_{\alpha \beta}(z-w)}{\left[(z-w)^{2}\right]^{\mu_{\alpha \beta}}}-\iota_{w, z} \frac{Q_{\alpha \beta}(z-w)}{\left[(z-w)^{2}\right]^{\mu_{\alpha \beta}}}\right) K, \\
u^{\alpha}(z) & =\sum_{n \in \mathbb{Z}} \sum_{m=0}^{\infty} \sum_{\sigma=1}^{\mathfrak{h}_{m}} u_{\{n, m, \sigma\}}^{\alpha}\left(z^{2}\right)^{n} h_{\sigma}^{(m)}(z)
\end{aligned}
$$


for $\alpha, \beta \in A$, where $\mu_{\alpha \beta}$ are positive integers, $K$ is the central element and $Q_{\alpha \beta}(z)$ are polynomials such that $Q_{\alpha \beta}(-z)=(-1)^{p_{\alpha} p_{\beta}} Q_{\beta \alpha}(z)$. Without lost of generality we can suppose that the leading (i. e. harmonic) term in the harmonic decomposition (1.5) for every $Q_{\alpha \beta}(z)=: p(z)$ is nonzero if $Q_{\alpha \beta}(z) \neq 0$. Then the right hand side of Eq. (5.2) uniquely determines the polynomials $Q_{\alpha \beta}(z)$. As a consequence of Eq. (5.2) (and 4.15) we have

$$
\left[(z-w)^{2}\right]^{\mu_{\alpha \beta}}\left[u^{\alpha}(z), u^{\beta}(w)\right]=0 .
$$

The Lie super algebra $\mathfrak{H}$ so obtained has a decomposition

$$
\begin{aligned}
\mathfrak{H} & =\mathfrak{H}_{\{+\}} \oplus \mathbb{C} K \oplus \mathfrak{H}_{\{-\}}, \\
\mathfrak{H}_{\{+\}} & =\operatorname{Span}_{\mathbb{C}}\left\{u_{\{n, m, \sigma\}}^{\alpha}: \alpha \in A, n \geqslant 0, m \in N \cup\{0\}, \sigma=1, \ldots, \mathfrak{h}_{m}\right\}, \\
\mathfrak{H}_{\{-\}} & =\operatorname{Span}_{\mathbb{C}}\left\{u_{\{n, m, \sigma\}}^{\alpha}: \alpha \in A, n<0, m \in N \cup\{0\}, \sigma=1, \ldots, \mathfrak{h}_{m}\right\} .
\end{aligned}
$$

Let $\mathcal{F}$ be the Fock representation space of $\mathfrak{H}$ determined by $\mathfrak{H}_{\{-\}}|0\rangle=0$ and $\left.K\right|_{\mathcal{F}}=$ $k \mathbb{I}$, where $|0\rangle \in \mathcal{F}$ is the Fock vacuum. Then the formal series 5.3 is represented on $\mathcal{F}$ as a field for every $\alpha \in A$. In fact we will prove more a general statement:

Proposition 5.1. Let a system of formal series (5.3) be given with coefficients $u_{\{n, m, \sigma\}}^{\alpha}$ generating a Lie superalgebra $\mathfrak{L}$ and such that the equation (5.4) is satisfied for some positive integers $\mu_{\alpha \beta}$ and all $\alpha, \beta \in A\left(u_{\{n, m, \sigma\}}^{\alpha}\right.$ are supposed to have a parity independent on $n, m$ and $\sigma$ ). Let $\mathfrak{L}_{\{+\}}$and $\mathfrak{L}_{\{-\}}$be the subalgebras of $\mathfrak{L}$ which are generated by the right hand sides of Eqs. (5.6) and (5.7), respectively. Let $\mathcal{U}$ be the representation of $\mathfrak{L}$ obtained by factorization of the universal enveloping algebra $U(\mathfrak{L})$ of $\mathfrak{L}$ by the left ideal $U(\mathfrak{L}) \mathfrak{L}_{\{-\}}$where $\mathfrak{L}$ is assumed to act by left multiplication. Then the formal series (5.3) is represented on $\mathcal{U}$ as a field for every $\alpha \in A$.

Proof. Let us denote the class of $a \in U(\mathfrak{L})$ in $\mathcal{U}$ by $[a]$. Thus we have to prove that

$$
u^{\alpha}(z)\left[u_{\left\{n_{1}, m_{1}, \sigma_{1}\right\}}^{\alpha_{1}} \cdots u_{\left\{n_{k}, m_{k}, \sigma_{k}\right\}}^{\alpha_{k}} \mathbb{I}\right] \in \mathcal{U} \llbracket z \rrbracket_{z^{2}}
$$

for all $k=0,1, \ldots$ and all values of the indices. We will make the proof by induction in $k$ : for $k=0$ Eq. 5.8 follows from the factorization by $\mathfrak{L}_{\{-\}}$. Suppose that 5.8 is satisfied for $k \geqslant 0$ and all values of the indices. Let us set

$$
v_{k}:=\left[u_{\left\{n_{1}, m_{1}, \sigma_{1}\right\}}^{\alpha_{1}} \cdots u_{\left\{n_{k}, m_{k}, \sigma_{k}\right\}}^{\alpha_{k}} \mathbb{I}\right] \in \mathcal{U} \text {. }
$$

Then we have to prove that $\left(z^{2}\right)^{N} u^{\alpha}(z) u_{\{n, m, \sigma\}}^{\beta} v_{k} \in \mathcal{U} \llbracket z \rrbracket$ for $N \gg 0$. By the inductive assumption $u_{\{n, m, \sigma\}}^{\beta} v_{k}=\left.P_{\{n+M, m, \sigma\}}\left(\partial_{w}\right)\left(w^{2}\right)^{M} u^{\beta}(w) v_{k}\right|_{w=0}$ for $M \gg 0$ (recall the definition (1.11) of $P_{\{n, m, \sigma\}}(z)$ ). Then by Lemma 2.3 for every $L \in \mathbb{N}$ there exist $N \in \mathbb{N}$ and a polynomial $Q(z, w) \in \mathbb{C}[z, w]$ such that

$$
\begin{gathered}
\left(z^{2}\right)^{N} u^{\alpha}(z) u_{\{n, m, \sigma\}}^{\beta} v_{k}= \\
=\left.\left[(z-w)^{2}\right]^{N} u^{\alpha}(z) P_{\{n+M, m, \sigma\}}\left(\partial_{w}\right)\left(w^{2}\right)^{M} u^{\beta}(w) v_{k}\right|_{w=0}= \\
=\left.Q\left(z-w, \partial_{w}\right)\left[(z-w)^{2}\right]^{L}\left(w^{2}\right)^{M} u^{\alpha}(z) u^{\beta}(w) v_{k}\right|_{w=0}
\end{gathered}
$$


for $M \gg 0$. On the other hand, it follows from 5.4 that $\left[(z-w)^{2}\right]^{L} u^{\alpha}(z) u^{\beta}(w) v_{k}$ $\in \mathcal{U} \llbracket z, w \rrbracket$ for $L \gg 0$ (as in the case of vertex algebras, after Def. 2.3. Consequently, $\left(z^{2}\right)^{N} u^{\alpha}(z) u_{\{n, m, \sigma\}}^{\beta} v_{k} \in \mathcal{U} \llbracket z \rrbracket$ for $N \gg 0$.

To obtain a vertex algebra structure we need additional assumptions.

Proposition 5.2. In the assumptions of Proposition 5.1 let us suppose that there exist even derivations $\mathrm{T}_{1}, \ldots, \mathrm{T}_{D}$ of $\mathfrak{L}$ such that

$$
\mathrm{T}_{\mu}\left(u^{\alpha}(z)\right)=\partial_{z^{\mu}}\left(u^{\alpha}(z)\right)
$$

for $\mu=1, \ldots, D$ and $\alpha \in A$. Then $\mathfrak{L}_{\{-\}}$is $\mathrm{T}$-invariant and hence $\mathrm{T}_{\mu}$ are represented on $\mathcal{U}$. Suppose also that $\mathcal{V}=\mathcal{U} / \mathcal{J}$ is a quotient representation of $\mathfrak{L}$ by a $\mathrm{T}$-invariant subrepresentation $\mathcal{J}$ such that the class $\hat{1}:=[\mathbb{I}] \in \mathcal{V}$ of $\mathbb{I} \in U(\mathfrak{L})$ is nonzero. Then if $\mathrm{T}_{\mu}$ are represented on $\mathcal{V}$ by $T_{\mu} \in$ End $\mathcal{V}$ it follows that the representation of the formal series (5.3) on $\mathcal{V}$ satisfy all the assumptions of the existence Theorem 4.1 and hence $\mathcal{V}$ has the structure of a vertex algebra.

Proof. Using Eqs. (1.20) one can prove that Eq. (5.9) is equivalent to

$$
\begin{aligned}
\mathrm{T}_{\mu}\left(u_{\{n, m, \sigma\}}^{\alpha}\right):= & \frac{n+m+\frac{D}{2}}{m+\frac{D}{2}} \sum_{\sigma_{1}=1}^{\mathfrak{h}_{m+1}} A_{\mu \sigma_{1} \sigma}^{(m+1)} u_{\left\{n, m+1, \sigma_{1}\right\}}^{\alpha}+ \\
& +\sum_{\sigma_{1}=1}^{\mathfrak{h}_{m-1}} 2(n+1) B_{\mu \sigma_{1} \sigma}^{(m-1)} u_{\left\{n+1, m-1, \sigma_{1}\right\}}^{\alpha} .
\end{aligned}
$$

Therefore, $\mathfrak{L}_{\{-\}}$is T-invariant. By Proposition $5.1 u^{\alpha}(z)$ acts as a field on $\mathcal{V}$ for every $\alpha \in A$. The verifications of the other assumptions of Theorem 4.1 are straightforward.

Corollary 5.3. The Fock space $\mathcal{F}$ defined above has the structure of a vertex algebra which is generated by the fields (5.3) satisfying the relations (5.2).

Proof. Eq. (5.10) and $\mathrm{T}_{\mu}(K)=0$ define an even derivation of the algebra $\mathfrak{H} 5.5$ since the relations [5.2) are $\partial$-invariant. To apply Propositions 5.1 and 5.2 we extend the system of formal series (5.3) with the constant series $K(z)=K$. Then the role of $\mathfrak{L}_{\{-\}}$is played by $\mathfrak{H}_{\{-\}}$and $\mathcal{F}$ is obtained by additional factorization of $\mathcal{U}$ by the subrepresentation generated by $K-k \mathbb{I}$ which is T-invariant. Finally, $\mathcal{F}$ is isomorphic to the symmetric superalgebra generated by $\mathfrak{H}_{\{+\}}$(5.6) so that the class $\widehat{1}$ is nonzero.

The vertex algebra obtained in Corollary 5.3 is called a free field vertex algebra. A Lie superalgebra $\mathfrak{L}$ and a system of series $[5.3$ satisfying the assumptions of Proposition 5.1 and possessing a system of even derivations $\mathrm{T}_{1}, \ldots, \mathrm{T}_{D}$, such that Eq. 5.9 holds is called a Lie superalgebra of formal distributions. 


\section{Categorical Properties of Vertex Algebras. Representations}

We begin with some basic categorical notions for vertex algebras in higher dimensions which are straightforward generalizations of the corresponding one from the chiral CFT [5]. A morphism $f$ of vertex algebras $\mathcal{V}$ and $\mathcal{V}^{\prime}$ is called a parity preserving linear map $f: \mathcal{V} \rightarrow \mathcal{V}^{\prime}$ such that

$$
\begin{aligned}
f\left(a_{\{n, m, \sigma\}} b\right) & =f(a)_{\{n, m, \sigma\}} f(b), \\
f\left(T_{\mu}(a)\right) & =T_{\mu}^{\prime}(f(a)), \\
f(\widehat{1}) & =f\left(\widehat{1}^{\prime}\right)
\end{aligned}
$$

for all $a, b \in \mathcal{V}, n \in \mathbb{Z}, m=0,1, \ldots, \sigma=1, \ldots, \mathfrak{h}_{m}$ and $\mu=1, \ldots, D$, where $T_{\mu}$, $\widehat{1}$ and $T_{\mu}^{\prime}, \widehat{1}^{\prime}$, are the translation endomorphisms and the vacuum, correspondingly in $\mathcal{V}$ and $\mathcal{V}^{\prime}$. An isomorphism of vertex algebras is a morphism which is an isomorphism as a linear map. An injective or surjecvtive morphism $f$ is such that the map $f$ is injective or surjective as a linear map, respectively. The image $g(\mathcal{U})$ and the kernel Ker $g$ of a morphism $g: \mathcal{U} \rightarrow \mathcal{V}$ are called a vertex subalgebra and ideal of $\mathcal{V}$, respectively. Note that the image $g(\mathcal{U})$ is itself a vertex algebra. If $f: \mathcal{V} \rightarrow \mathcal{V}^{\prime}$ is a surjective morphism and $\mathcal{J}$ is its kernel then the quotient space $\mathcal{V} / \mathcal{J}$ possesses the structure of a vertex algebra isomorphic to $\mathcal{V}^{\prime}$. It is called a quotient vertex algebra.

Proposition 6.1. Let $\mathcal{V}$ be a vertex algebra.

(a) A super-subspace $\mathcal{U}$ of $\mathcal{V}$ has the structure of a vertex subalgebra of $\mathcal{V}$ under the inclusion $\mathcal{U} \hookrightarrow \mathcal{V}$ iff $\widehat{1} \in \mathcal{U}$ and $a_{\{n, m, \sigma\}} b \in \mathcal{U}$ for all $a, b \in \mathcal{U}$ and $n \in \mathbb{Z}$, $m=0,1, \ldots, \sigma=1, \ldots \mathfrak{h}_{m}$.

(b) A super-subspace $\mathcal{J}$ of $\mathcal{V}$ is an ideal iff $\mathcal{J}$ is $T_{\mu}$-invariant $(\mu=1, \ldots, D), \widehat{1} \notin \mathcal{J}$ and $a_{\{n, m, \sigma\}} b \in \mathcal{J}$ for all $a \in \mathcal{V}, b \in \mathcal{J}$ and $n \in \mathbb{Z}, m=0,1, \ldots, \sigma=1, \ldots \mathfrak{h}_{m}$.

Proof. To prove the statement (a) first observe that $\mathcal{U}$ is $T_{\mu}$-invariant $(\mu=1, \ldots, D)$, since for every $a \in \mathcal{U}: a=\left.Y(a, z) \widehat{1}\right|_{z=0}$ and then $T_{\mu} a=\left.\partial_{z^{\mu}}(Y(a, z) \widehat{1})\right|_{z=0}$. Then (a) follows directly by the above definitions. For the proof of part (b), as in the chiral CFT ([5]), we need to show that $a_{\{n, m, \sigma\}} b \in \mathcal{J}$ for all $a \in \mathcal{J}, b \in \mathcal{V}, n \in$ $\mathbb{Z}, m=0,1, \ldots, \sigma=1, \ldots \mathfrak{h}_{m}$. However the last property is a consequence of the quasisymmetry relation:

$$
Y(a, z) b=(-1)^{p_{a} p_{b}} e^{z \cdot T} Y(b,-z) a
$$

(here $z \cdot T=\sum_{\mu=1}^{D} a^{\mu} T_{\mu}$ ) for all $a, b \in \mathcal{V}$ (the right hand side of Eq. (6.4) is understood as an action of the series $e^{z \cdot T} \in(E n d \mathcal{V}) \llbracket z \rrbracket$ on a series belonging to $\left.\mathcal{V} \llbracket z \rrbracket z^{2}\right)$.

Here is a sketch of the proof of Eq. 6.4): using the series $\mathcal{Y}_{n}$ introduced in Proposition 4.2 we first derive that $\mathcal{Y}_{2}(a, z, b, w ; \widehat{1}) \in \mathcal{V} \llbracket z, w \rrbracket_{(z-w)^{2}}$ and

$$
\begin{gathered}
\left.\mathcal{Y}_{2}(a, z ; b, w ; \widehat{1})\right|_{w=0}=\mathcal{Y}_{1}(a, z ; b)=Y(a, z) b, \\
e^{w \cdot T} \mathcal{Y}_{2}\left(a, z_{1} ; b, z_{2} ; \widehat{1}\right)=\mathcal{Y}_{2}\left(a, z_{1}+w ; b, z_{2}+w ; \widehat{1}\right)
\end{gathered}
$$

(using the argument of the proof of Proposition $3.2(a) \Rightarrow(b)$ ); we then apply to the left hand side of Eq. (6.6) the $\mathbb{Z}_{2}$-symmetry (4.17) and set $z_{1}:=0, w=-z_{2}:=z$. 
Let $\mathcal{V}$ be a vertex algebra over $\mathbb{C}^{D}$ and let $A: \mathbb{C}^{D^{\prime}} \rightarrow \mathbb{C}^{D}$ be a linear orthogonal map $((A x) \cdot(A x)=x \cdot x)$ with a matrix $\left(A_{\nu}^{\mu}\right): A e_{\nu}^{\prime}=\sum_{\nu=1}^{D} A_{\nu}^{\mu} e_{\mu}$ in the standard bases $\left\{e_{\mu}\right\}_{\mu=1}^{D}$ and $\left\{e_{\nu}^{\prime}\right\}_{\nu=1}^{D^{\prime}}\left(D^{\prime} \leqslant D\right)$. Then the formal series $Y^{\prime}(a, x):=$ $\left.Y(a, z)\right|_{z=A x}$ for $x=\left(x^{1}, \ldots, x^{D^{\prime}}\right)$ and $(A x)^{\mu}=\sum_{\nu=1}^{D^{\prime}} A_{\nu}^{\mu} x^{\nu}$, are correctly defined for every $a \in \mathcal{V}$ as series belonging to $(E n d \mathcal{V}) \llbracket x, 1 / x^{2} \rrbracket$. They generate, combined with the maps $T_{\nu}^{\prime}:=\sum_{\mu=1}^{D} A_{\nu}^{\mu} T_{\mu}$ for $\nu=1, \ldots, D^{\prime}$, a structure of vertex algebra on $\mathcal{V}$ over $\mathbb{C}^{D^{\prime}}$ with the same $\mathbb{Z}_{2}$-grading and vacuum $\hat{1} \in \mathcal{V}$. We denote this vertex algebra by $A^{*} \mathcal{V}$ and call it a restriction of $\mathcal{V}$ over $\mathbb{C}^{D^{\prime}}$.

Let $\mathcal{V}$ and $\mathcal{V}^{\prime}$ be vertex algebras over $\mathbb{C}^{D}$ and let the corresponding state-field correspondence, translation operators and vacua be: $Y(a, z), T_{\mu}, \widehat{1}$ (in $\mathcal{V}$ ) and $Y^{\prime}(b, x)$, $T_{\nu}^{\prime}, \widehat{1}^{\prime}$ (in $\mathcal{V}^{\prime}$ ). Then for every $a \in \mathcal{V}$ and $b \in \mathcal{V}^{\prime}$ the formal series $Y\left(a, z_{1}\right) \otimes$ $Y^{\prime}\left(b, z_{2}\right)$ is a field on the superspace $\mathcal{V} \otimes \mathcal{V}^{\prime}$ and consequently one can define the

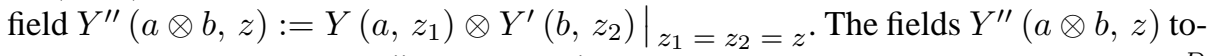
gether with the operators $T_{\mu}^{\prime \prime}:=T_{\mu}+T_{\mu}^{\prime}$ generate a vertex algebra structure over $\mathbb{C}^{D}$ on $\mathcal{V} \otimes \mathcal{V}^{\prime}$ with a vacuum $\widehat{1} \otimes \widehat{1}^{\prime}$. This vertex algebra is called a tensor product of $\mathcal{V}$ and $\mathcal{V}^{\prime}$ and we will denote it by $\mathcal{V} \otimes \mathcal{V}^{\prime}$.

A representation of the vertex algebra $\mathcal{V}$ is called a super space $\mathcal{M}$ together with a parity preserving linear map $\mathcal{V} \rightarrow($ End $\mathcal{M}) \llbracket z, 1 / z^{2 \rrbracket}: a \mapsto Y_{\mathcal{M}}(a, z)$ and even endomorphisms $T_{\mu} \in$ End $\mathcal{M}$ for $\mu=1, \ldots, D$ called again translation endomorphisms such that:

(a) $Y_{\mathcal{M}}(a, z)$ is a field and $Y_{\mathcal{M}}(a, z), Y_{\mathcal{M}}(b, z)$ are mutually local for all $a$ and $b \in \mathcal{V}$

(b) $Y_{\mathcal{M}}(a, z)_{\{n, m, \sigma\}} Y_{\mathcal{M}}(b, z)=Y_{\mathcal{M}}\left(a_{\{n, m, \sigma\}} b, z\right)$ for all $a, b \in \mathcal{V}$ and all $n \in \mathbb{Z}, m=0,1, \ldots, \sigma=1, \ldots, \mathfrak{h}_{m}$, where the field $\{n, m, \sigma\}$-products are defined in accord with Theorem 2.1.

(c) $\left[T_{\mu}, Y_{\mathcal{M}}(a, z)\right]=\partial_{z^{\mu}} Y_{\mathcal{M}}(a, z)$ for $\mu=1, \ldots, D$.

An example of a representation of a vertex algebra $\mathcal{V}$ is provided by the vertex algebra itself and it is called the vacuum representation of $\mathcal{V}$.

\section{Conformal Vertex Algebras}

We begin by recalling some basic facts about the conformal group and its action for higher space dimensions $D$. We will use the complex conformal group $\mathfrak{C}_{\mathbb{C}}$ which is convenient to choose to be the connected complex spinor group $\operatorname{Spin}_{0}(D+2 ; \mathbb{C})=: \mathfrak{C}_{\mathbb{C}}$ (the last is in fact a covering of the "geometrical" conformal group in space dimensions $D \geqslant 3$ ). The geometrical action of $\mathfrak{C}_{\mathbb{C}}$ on $\mathbb{C}^{D}$ will be denoted as

$$
\mathbb{C}^{D} \ni z \mapsto g(z) \in \mathbb{C}^{D} \quad\left(g \in \mathfrak{C}_{\mathbb{C}}\right)
$$

and it generally has singularities: we will denote the regularity of $z$ for $g$ as " $g(z) \in$ $\mathbb{C}^{D}$ ". The Lie algebra $\mathfrak{c}_{\mathbb{C}}$ of $\mathfrak{C}_{\mathbb{C}}$ is isomorphic to so $(D+2 ; \mathbb{C})$ and it has generators 
$T_{1}, \ldots, T_{D}, S_{1}, \ldots, S_{D}, H$ and $\Omega_{\mu \nu}$ for $1 \leqslant \mu<\nu \leqslant D\left(\Omega_{\nu \mu}:=-\Omega_{\mu \nu}\right)$, with the following commutation relations:

$$
\begin{aligned}
{\left[H, \Omega_{\mu \nu}\right] } & =0=\left[T_{\mu}, T_{\nu}\right]=\left[S_{\mu}, S_{\nu}\right] \\
{\left[\Omega_{\mu_{1} \nu_{1}}, \Omega_{\mu_{2} \nu_{2}}\right] } & =\delta_{\mu_{1} \mu_{2}} \Omega_{\nu_{1} \nu_{2}}+\delta_{\nu_{1} \nu_{2}} \Omega_{\mu_{1} \mu_{2}}-\delta_{\mu_{1} \nu_{2}} \Omega_{\nu_{1} \mu_{2}}-\delta_{\nu_{1} \mu_{2}} \Omega_{\mu_{1} \nu_{2}} \\
{\left[H, T_{\mu}\right] } & =T_{\mu}, \quad\left[H, S_{\mu}\right]=-S_{\mu} \\
{\left[\Omega_{\mu \nu}, T_{\rho}\right] } & =\delta_{\mu \rho} T_{\nu}-\delta_{\nu \rho} T_{\mu},\left[\Omega_{\mu \nu}, S_{\rho}\right]=\delta_{\mu \rho} S_{\nu}-\delta_{\nu \rho} S_{\mu} \\
{\left[S_{\mu}, T_{\nu}\right] } & =2 \delta_{\mu \nu} H+2 \Omega_{\mu \nu} .
\end{aligned}
$$

Thus: $T_{1}, \ldots, T_{D}$ are the generators of the translations on $\mathbb{C}^{D}, t_{a}(z):=z+a$, $t_{a}=e^{a \cdot T}, a \cdot T \equiv \sum_{\mu=1}^{D} a^{\mu} T_{\mu} ; H$ is the generator of the dilations $e^{\lambda H}(z)=e^{\lambda} z ;$ $\Omega_{\mu \nu}$ are the generators of the orthogonal group of $\mathbb{C}^{D}$ ( $e^{\vartheta \Omega_{\mu \nu}}$ being the rotation on angle $\vartheta$ in the plane $(\mu, \nu)$ of $\mathbb{R}^{D}$; and finally, $S_{\mu}$ are generator of the special conformal transformations:

$$
s_{a}:=e^{a \cdot S}, \quad s_{a}(z)=\frac{z+z^{2} a}{1+2 a \cdot z+a^{2} z^{2}} \quad\left(a, z \in \mathbb{C}^{D}\right) .
$$

(For the explicit expression of the generators $T_{1}, \ldots, T_{D}, S_{1}, \ldots, S_{D}, H$ and $\Omega_{\mu \nu}$ in terms of the standard generators of $s o(D+2 ; \mathbb{C})-$ see for example [2].) We will call the Lie subalgebra of $\mathfrak{c}_{\mathbb{C}}$ generated by $\Omega_{\mu \nu}(1 \leqslant \mu<\nu \leqslant D)$ the rotation subalgebra $(\cong s o(D ; \mathbb{C}))$ and its corresponding subgroup in $\mathfrak{C}_{\mathbb{C}}-$ the spinor rotation subgroup $\left(\cong \operatorname{Spin}_{0}(D ; \mathbb{C})\right)$.

An important element of the group $\mathfrak{C}_{\mathbb{C}}$ is the Weyl inversion $j_{W}$ :

$$
j_{W}(z):=\frac{R_{D}(z)}{z^{2}}
$$

where $R_{\mu}(z)$ for $z=\left(z^{1}, \ldots, z^{D}\right)$ and $\mu=1, \ldots, D$, is the reflection

$$
R_{\mu}\left(z^{1}, \ldots, z^{D}\right)=\left(z^{1}, \ldots,-z^{\mu}, \ldots, z^{D}\right)
$$

$j_{W}$ is represented in $S O(D+1,1 ; \mathbb{R})$ as a rotation on $\pi$ in the plane $(D, D+1)$, i. e., $j_{W}=e^{\pi\left(T_{D}+S_{D}\right)}$. Note that $j_{W}^{2}(z)=z$ for all $z$ but nevertheless $j_{W}^{2}$ as an element of $\operatorname{Spin}_{0}(D+2, \mathbb{C})$ is the nonunit central element $C=-\mathbb{I}_{\text {Cliff }}$,

$$
j_{W}^{2}=C
$$

where $\mathbb{I}_{\text {Cliff }}$ is the Clifford algebra unit.

The passage from the vertex algebras to the globally conformal invariant QFT needs first an additional symmetry structure for our vertex algebras. For this purpose we will extend the abelian Lie algebra of the translations $T_{1}, \ldots, T_{D}$ to the conformal one $\mathfrak{c}_{\mathbb{C}} \cong s o(D+2 ; \mathbb{C})$. 
Definition 7.1. A conformal vertex algebra is called a vertex algebra $\mathcal{V}$ endowed with an action of $\mathfrak{c}_{\mathbb{C}}$ by even linear automorphisms such that

$$
\begin{aligned}
{[H, Y(a, z)]=} & z \cdot \partial_{z} Y(a, z)+Y(H a, z) \\
{\left[\Omega_{\mu \nu}, Y(a, z)\right]=} & z^{\mu} \partial_{z^{\nu}} Y(a, z)-z^{\nu} \partial_{z^{\mu}} Y(a, z)+Y\left(\Omega_{\mu \nu} a, z\right) \\
{\left[S_{\mu}, Y(a, z)\right]=} & \left(-z^{2} \partial_{z^{\mu}}+2 z^{\mu} z \cdot \partial_{z}\right) Y(a, z)+2 z^{\mu} Y(H a, z)+ \\
& +2 \sum_{\nu=1}^{D} z^{\nu} Y\left(\Omega_{\nu \mu} a, z\right)+Y\left(S_{\mu} a, z\right)
\end{aligned}
$$

$\left(z \cdot \partial_{z} \equiv \sum_{\mu=1}^{D} z^{\mu} \partial_{z^{\mu}}, z^{2} \equiv z \cdot z \equiv \sum_{\mu=1}^{D} z^{\mu} z^{\mu}\right)$. The compatibility of the commutation relations (7.2) with Eqs. (7.7)-7.9) is obtained by a straightforward computation. We require also that:

(a) the enodomorphism $H$ is diagonalizable with nonnegative eigenvalues (the energy positivity condition).

(b) The representation of the rotation subalgebra so $(D ; \mathbb{C}) \subset \mathfrak{c}_{\mathbb{C}}$ on $\mathcal{V}$ decomposes into a direct sum of finite dimensional irreducible subrepresentations. Then this representation can admit integration to an action of the spinor rotation subgroup $\operatorname{Spin}_{0}(D ; \mathbb{C})$.

(c) Let $C$ be the central element (7.6) $\left(C^{2}=\mathbb{I}\right)$ then $H+\frac{1}{4}(\mathbb{I}-C)$ has an integer spectrum. In particular, $H$ has only integer or half-integer eigenvalues.

(d) The vacuum $\hat{1}$ is the only one $\mathfrak{c}_{\mathbb{C}}$-invariant element of $\mathcal{V}$ up to multiplication, i. e., $X a=0$ for every $X \in \mathfrak{c}_{\mathbb{C}} \Leftrightarrow a \sim \widehat{1}$.

If $a \in \mathcal{V}$ is an eigenvector of $H$ we will denote its eigenvalue by $w t_{H}(a)$ and call it weight of $a$ :

$$
H a=w t_{H}(a) a .
$$

Then if $a, b \in \mathcal{V}$ have fixed weights:

$$
w t_{H}\left(a_{\{n, m, \sigma\}} b\right)=w t_{H}(a)+w t_{H}(b)+2 n+m,
$$

which follows from the equation

$$
a_{\{n, m, \sigma\}} b=\left.P_{\{n+N, m, \sigma\}}\left(\partial_{z}\right)\left(z^{2}\right)^{N} Y(a, z) b\right|_{z=0}
$$

for $N \gg 0$ (see Eq. (1.11) and the relation (7.7).

As a consequence of Definition $7.1(a)$ and $(d)$, and the commutation relations 7.2 the endomorphisms $S_{\mu}$ will have a nilpotent action on a conformal vertex algebra $\mathcal{V}$. Thus the representation of $S_{1}, \ldots, S_{D}, H$ and $\Omega_{\mu \nu}(1 \leqslant \mu<\nu \leqslant D)$ on $\mathcal{V}$ can be integrated to a group action. Recall that the last generators span a Lie subalgebra $=: \mathfrak{c}_{\mathbb{C}, 0}$ of $\mathfrak{c}_{\mathbb{C}}$ which corresponds to the subgroup $\mathfrak{C}_{\mathbb{C}, 0}$ of $\mathfrak{C}_{\mathbb{C}}-$ the connected part of the stabilizer of $0 \in \mathbb{C}^{D}$. Note that $\mathfrak{C}_{\mathbb{C}, 0}$ is isomorphic to the inhomogeneous connected spinor group of $\mathbb{C}^{D}$ with dilations (i. e. the complex Euclidean spinor group with dilations) and it is simply connected. Denote the obtained action by

$$
\pi_{0}: \mathfrak{C}_{\mathbb{C}, 0} \rightarrow A u t_{0} \mathcal{V}
$$


and define

$$
\pi_{z}(g):=\pi_{0}\left(t_{g(z)}^{-1} g t_{z}\right)
$$

for all pairs $(z, g)$ in some neighbourhood of $(0, \mathbb{I}) \in \mathbb{C}^{D} \times \mathfrak{C}_{\mathbb{C}}$ such that $g(z) \in \mathbb{C}^{D}$. Note that $t_{g(z)}^{-1} g t_{z}(0)=0$ if $z$ is regular for $g$ (i. e., $g(z) \in \mathbb{C}^{D}$ ) so that $t_{g(z)}^{-1} g t_{z}(0) \in$ $\mathfrak{C}_{\mathbb{C}, 0}$ for small $z$ and $g$.

Proposition 7.1. (a) The function $\pi_{z}(g)$ (7.13) is rational in $z$ for every $g \in \mathfrak{C}_{\mathbb{C}}$ with values in $(E n d \mathcal{V})_{0}$, i. e. it can be expressed as a ratio of a polynomial belonging to $(\text { End } \mathcal{V})_{0}[z]$ and a polynomial belonging to $\mathbb{C}[z]$. It has the cocycle property

$$
\pi_{z}\left(g_{1} g_{2}\right)=\pi_{g_{2}(z)}\left(g_{1}\right) \pi_{z}\left(g_{2}\right) \quad \text { iff } \quad g_{1} g_{2}(z), g_{2}(z) \in \mathbb{C}^{D}
$$

and satisfies $\pi_{z}\left(t_{a}\right)=\mathbb{I}$.

(b) Let the assumptions of Definition 7.1 are supposed except the condition (c). Then if the cocycle $\pi_{z}(g)$ (7.13) possesses a continuation to a rational function in $z$ it follows that the condition (c) of Definition 7.1 is also satisfied.

Proof. (a) Eq. (7.14) is a straightforward consequence of (7.13) for small $z, g_{1}$ and $g_{2}$. If $g$ belongs to the (spinor) Euclidean group of $\mathbb{C}^{D}$ with dilations then $t_{g(z)}^{-1} g t_{z}=g_{1}$ does not depend on $z$ and it is just the homogeneous part of $g$ (i. e. the projection on the spinor and dilation group). Thus if we prove the rationality of $\pi_{z}\left(j_{W}\right)$ it will follow for the general $\pi_{z}(g)$ because of Eq. (7.14) and the fact the conformal group $\mathfrak{C}_{\mathbb{C}}$ is generated by $j_{W}$ and the spinor Euclidean group with dilations. To compute $\pi_{z}\left(j_{W}\right)$ first we observe that

$$
t_{j_{W}(z)}^{-1} j_{W} t_{z}=s_{-R_{D}(z)} O, \quad O_{z}(w)=\frac{1}{z^{2}} \sum_{\mu=1}^{D} \frac{\left(R_{D}(z)\right)^{\mu}}{\sqrt{z^{2}}} R_{\mu}\left(R_{D}(w)\right)
$$

where $s_{-R_{D}(z)}, O_{z} \in \mathfrak{C}_{\mathbb{C}, 0}$ so that

$$
\pi_{z}\left(j_{W}\right)=\pi_{0}\left(s_{-R_{D}(z)}\right) \pi_{0}\left(O_{z}\right) .
$$

Then $\pi_{0}\left(O_{z}\right)$ is rational due to Definition 7.1 $(c)$ : indeed, if $\pi_{0}$ is a subrepresentation of the spinor representation of the Clifford algebra Cliff $(D ; \mathbb{R})$ (for even $D$ it is uniquely determined and for odd $D$, there are two irreducible representations, up to equivalence), then $O_{z}$ is represented by $\pm\left(z^{2}\right)^{-H-\frac{1}{2}} \sum_{\mu=1}^{D} z^{\mu} \gamma_{D} \gamma_{\mu}$ ), where $\gamma_{\mu}$ are the Clifford algebra generators; in the general case $\pi_{0}$ is a direct sum of subrepresentations of some tensor power of the above one.

To prove the second part (b) we observe that the last argument is invertible: the rationality of $\pi_{0}\left(O_{z}\right)$ implies the condition $(c)$ of Definition 7.1

A standard consequence of the commutation relations (7.2) is that the full eigenspaces of the action of $H$ on a conformal vertex algebra are invariant for the action of the rotation subalgebra $s o(D ; \mathbb{C})$. Therefore, the irreducible subrepresentations of so $(D ; \mathbb{C})$ are eigenspaces for $H$.

Lemma 7.2. For every element $a$ in a conformal vertex algebra $\mathcal{V}$ there exists a finite dimensional subspace $\mathcal{U}$ of $\mathcal{V}$ which contains a and is invariant with respect to the representation $\pi_{0}$ (7.12). Therefore, $\mathcal{U}$ is also invariant for the cocycle $\pi_{z}(g)$ (7.13). 
Proof. Because of Definition $7.1(b)$ it is sufficient to prove the lemma in the case when $a$ belongs to some irreducible subrepresentation $\mathcal{U}_{0}$ of the rotation group. Then $\left.H\right|_{\mathcal{U}_{0}}=d \mathbb{I}$ for $2 d \in\{0\} \cup \mathbb{N}$. Therefore, $S_{\mu_{1}} \ldots S_{\mu_{2 d}} \mathcal{U}_{0}=0$ for all $\mu_{k}=1, \ldots, D$ $(k=1, \ldots, 2 d)$ and we can set then the space $\mathcal{U}$ to be the linear span of all vectors belonging to $S_{\mu_{1}} \ldots S_{\mu_{k}} \mathcal{U}_{0}(k=0, \ldots, 2 d-1)$.

Let $f(\lambda)$ and $f^{\prime}\left(\lambda, \lambda^{\prime}\right)$ be functions with values in a finite dimensional vector space $V$ which are holomorphic in $\lambda$ and $\lambda^{\prime}$ in a neighebouhood of $0 \in \mathbb{C}$. Then we set $\iota_{\lambda} f(\lambda) \in V \llbracket \lambda \rrbracket$ and $\iota_{\lambda, \lambda^{\prime}} f^{\prime}\left(\lambda, \lambda^{\prime}\right) \in V \llbracket \lambda, \lambda^{\prime} \rrbracket$ to be just the Taylor series of $f$ and $f^{\prime}$ around $0 \in \mathbb{C}$. This definition is applicable to the functions $\pi_{z}\left(e^{\lambda X}\right)$ and $\pi_{e^{\lambda^{\prime} X^{\prime}(z)}}\left(e^{\lambda X}\right)$ for $X, X^{\prime} \in \mathfrak{c}_{\mathbb{C}}$ because of Lemma7.2 Then Eq. (7.14) implies

$$
\iota_{\lambda, \lambda^{\prime}}\left(\pi_{z}\left(e^{\lambda X} e^{\lambda^{\prime} X^{\prime}}\right)\right)=\iota_{\lambda, \lambda^{\prime}}\left(\pi_{e \lambda X}(z)\left(e^{\lambda^{\prime} X^{\prime}}\right)\right) \iota_{\lambda, \lambda^{\prime}}\left(\pi_{z}\left(e^{\lambda X}\right)\right) .
$$

We will distinct the above notations $\iota_{\lambda}$ and $\iota_{\lambda, \lambda^{\prime}}$ from the similar $\iota_{z_{1}}, \ldots, z_{n}$ from Sect. [4 by the type of the arguments $\lambda, \lambda^{\prime}$ and $z_{k}$.

It follows from the above constructions that the following equation is valid for any conformal vertex algebra $\mathcal{V}$ :

$$
e^{\lambda X} Y(a, z) e^{-\lambda X} b=Y\left(\iota_{\lambda} \pi_{z}\left(e^{\lambda X}\right) a, e^{\lambda X}(z)\right) b
$$

as series belonging to $\mathcal{V} \llbracket z \rrbracket_{z^{2}} \llbracket \lambda \rrbracket$ for all $a, b \in \mathcal{V}$ and $X \in \mathfrak{c}_{\mathbb{C}}$. In the case of $X \in \mathfrak{c}_{\mathbb{C}, 0}$ Eq. (7.18) follows from the construction of $\pi_{0}$ (7.12) and relations (7.7)-(7.9). For $X=T_{\mu},(7.12)$ is just a consequence of Eq. (3.1).

\section{Hermitean Structure in Conformal Vertex Algebras}

Besides the conformal structure the passage from the vertex algebras to the QFT requires a Hermitean structure. In this section we will use besides the variables $z, w$, etc., their $D$-dimensional conjugate variables $\bar{z} \equiv \overline{\left(z^{1}, \ldots, z^{D}\right)}=\left(\overline{z_{1}}, \ldots, \overline{z_{D}}\right)$, etc. If $z$ is considered as a formal variable then $\bar{z}$ will be treated as an independent formal variable and if $z$ takes values in $\mathbb{C}^{D}$ then $\bar{z}$ will be its complex conjugate. We set also

$$
\overline{u(z)}=\bar{u}(\bar{z}), \quad \overline{\bar{z}}=z
$$

for a series $u(z) \in \mathbb{C} \llbracket z, 1 / z^{2} \rrbracket$, where $\bar{u}(z)$ stands for the series with complex conjugate coefficients.

Define the following conjugation

$$
z \mapsto z^{*}:=\frac{\bar{z}}{\bar{z}^{2}} .
$$

It can be written also by the Weyl reflection (7.4) as:

$$
z^{*}=j_{W}\left(R_{D}(\bar{z})\right) \equiv j_{W}^{-1}\left(R_{D}(\bar{z})\right) .
$$

The corresponding conjugations on the complex conformal Lie algebra and group are introduced by:

$$
\begin{gathered}
\left(T_{\mu}\right)^{*}:=-S_{\mu} \quad(1 \leqslant \mu \leqslant D) \\
H^{*}=-H, \quad \Omega_{\mu \nu}^{*}=\Omega_{\mu \nu} \quad(1 \leqslant \mu<\nu \leqslant D) \\
\left(e^{\lambda X}\right)^{*}=e^{\bar{\lambda} X^{*}} \text { for } \quad X \in \mathfrak{c}_{\mathbb{C}} \text { and } \lambda \in \mathbb{C}
\end{gathered}
$$


as $\left[X_{1}, X_{2}\right]^{*}=\left[X_{1}^{*}, X_{2}^{*}\right]$ and $g_{1}^{*} g_{2}^{*}=\left(g_{1} g_{2}\right)^{*}$ for $X_{1}, X_{2} \in \mathfrak{c}_{\mathbb{C}}$ and $g_{1}, g_{2} \in \mathfrak{C}_{\mathbb{C}}$. Since the conjugation $*$ uses the conformal inversion $z \mapsto \frac{z}{z^{2}}$ under which the generators $T_{\mu}$ and $S_{\mu}$ are conjugated, we have the consistency relation:

$$
g(z)^{*}=g^{*}\left(z^{*}\right)
$$

for all $g \in \mathfrak{C}_{\mathbb{C}}$ and $z \in \mathbb{C}^{D}$ such that $z^{*}, g(z) \in \mathbb{C}^{D}$.

For a series $a(z) \in V \llbracket z, 1 / z^{2} \rrbracket, V$ being a complex vector space, the substitution $a\left(z^{*}\right)$ is correctly defined by Eq. 1.15 as the series

$$
a\left(z^{*}\right):=\left.J[a(w)]\right|_{w=\bar{z}} \in V \llbracket \bar{z}, 1 / \bar{z}^{2} \rrbracket .
$$

If $V$ is endowed with a Hermitean form $\langle a \mid b\rangle \in \mathbb{C}$ for $a, b \in V$, then we will use the convention:

$$
\langle a \mid u(z) b\rangle=\langle\bar{u}(\bar{z}) a \mid b\rangle=u(z)\langle a \mid b\rangle \quad(\langle a \mid b\rangle=\overline{\langle b \mid a\rangle})
$$

for a series $u(z) \in \mathbb{C} \llbracket z, 1 / z^{2} \rrbracket$.

Definition 8.1. A conformal vertex algebra $\mathcal{V}$ with Hermitean structure is a conformal vertex algebra equipped with a nondegenerate Hermitean form $\langle a \mid b\rangle \in \mathbb{C}$ for $a, b \in$ $\mathcal{V}$, compatible with the $Z_{2}$-grading of $\mathcal{V}\left(\langle a \mid b\rangle=0\right.$ if $a \in \mathcal{V}_{0}$ and $\left.b \in \mathcal{V}_{1}\right)$ and possessing an antilinear even involution $a \mapsto a^{+}\left(a \in \mathcal{V} ;(\lambda a)^{+}=\bar{\lambda} a, a^{++}=a\right.$, $\left.\left(\mathcal{V}_{0,1}\right)^{+}=\mathcal{V}_{0,1}\right)$ satisfying the following conditions

$$
\begin{gathered}
\langle a \mid X b\rangle=-\left\langle X^{*} a \mid b\right\rangle, \\
\left\langle a \mid Y\left(c^{+}, z\right) b\right\rangle=\overline{\left\langle b \mid Y\left(\pi_{z^{*}}\left(j_{W}\right)^{-1} c, z^{*}\right) a\right\rangle}
\end{gathered}
$$

for all $a, b, c \in \mathcal{V}$ and $X \in \mathfrak{c}_{\mathbb{C}}$. Here the last equality is understood in the sense of rational functions in $z$ and it is correct in view of the following remark.

Remark 8.1. (a) As a consequence of Eq. (7.11) and the orhogonality of the different eigenspaces of $H$ (because of 8.7 and 8.3 ) we have

$$
\langle a \mid Y(c, z) b\rangle \in \mathbb{C}\left[z, 1 / z^{2}\right] \equiv \mathbb{C}[z] z^{2},
$$

(note that in accord with Definition 2.1 we only have $\langle a \mid Y(c, z) b\rangle \in \mathbb{C} \llbracket z \rrbracket z^{2}$ ). Note also that due to the decomposition (7.16) we will also have

$$
\pi_{z}\left(j_{W}^{ \pm 1}\right) a \in \mathcal{V}\left[z, 1 / z^{2}\right] .
$$

(b) The conjugation in Eq. 8.7) is a combination of the "Minkowski" conjugation $z \mapsto$ $R_{D}(\bar{z})$ and the Weyl reflection since it can be rewritten (using Eq. 8.2) also as

$$
\left\langle a \mid Y\left(c^{+}, z\right) b\right\rangle=\left\langle Y\left(\pi_{R_{D}(\bar{z})}\left(j_{W}^{-1}\right) c, j_{W}^{-1}\left(R_{D}(\bar{z})\right)\right) a \mid b\right\rangle .
$$

This conjugation law is also idempotent as a consequence of Eq. 8.19 below. 
Proposition 8.1. In any conformal vertex algebra $\mathcal{V}$ endowed with a Hermitean form $\langle a \mid b\rangle \in \mathbb{C}$ for $a, b \in \mathcal{V}$, satisfying Eqs. [8.7) and [8.9), the correlation functions $\left\langle b \mid Y\left(a_{1}, z_{1}\right) \ldots Y\left(a_{n}, z_{n}\right) c\right\rangle$ are rational for all $a_{1}, \ldots, a_{n}, b, c \in \mathcal{V}$ in the sense that they belong to $\iota_{z_{1}, \ldots, z_{n}}\left(\mathbb{C}\left[z_{1}, \ldots, z_{n}\right]_{R_{n}}\right)$ (see Sect. 4). In particular, they are regular for $z_{k}^{2} \neq 0$ and $\left(z_{k}-z_{l}\right)^{2} \neq 0(k, l=1, \ldots, n)$. The vacuum correlation functions

$$
\mathcal{W}_{n}\left(a_{1}, \ldots, a_{n} ; z_{1}, \ldots, z_{n}\right):=\iota_{z_{1}, \ldots, z_{n}}^{-1}\left\langle\widehat{1} \mid Y\left(a_{1}, z_{1}\right) \ldots Y\left(a_{n}, z_{n}\right) \hat{1}\right\rangle
$$

are globally conformal invariant in the sense that

$$
\begin{gathered}
\mathcal{W}_{n}\left(\pi_{z_{1}}(g) a_{1}, \ldots, \pi_{z_{n}}(g) a_{n} ; g\left(z_{1}\right), \ldots, g\left(z_{n}\right)\right)= \\
=\mathcal{W}_{n}\left(a_{1}, \ldots, a_{n} ; z_{1}, \ldots, z_{n}\right)
\end{gathered}
$$

(for all $g \in \mathfrak{C}_{\mathbb{C}}$ ) as rational functions in $z_{1}, \ldots, z_{n}$.

Proof. It follows from locality and Eq. 8.9 that

$$
\left(\prod_{k=1}^{n} z_{k}^{2}\right)^{N}\left(\prod_{1 \leqslant l<m \leqslant n} z_{l m}^{2}\right)^{N}\left\langle b \mid Y\left(a_{1}, z_{1}\right) \ldots Y\left(a_{n}, z_{n}\right) c\right\rangle \in \mathbb{C}\left[z_{1}, \ldots, z_{n}\right]
$$

for $N \gg 0\left(z_{l m}:=z_{l}-z_{m}\right)$. Then we multiply by

$$
\iota_{z_{1}, \ldots, z_{n}}\left(\prod_{k=1}^{n} z_{k}^{2}\right)^{-N}\left(\prod_{1 \leqslant l<m \leqslant n} z_{l m}^{2}\right)^{-N}
$$

as in the proof of Proposition 4.2 To prove Eq. $\frac{8.13}{3}$ we first obtain by Eq. (7.18) and by the conformal invariance of the vacuum $(\operatorname{Def} . \overline{7.1}(d))$ that

$$
\begin{gathered}
e^{\lambda X} Y\left(a_{1}, z_{1}\right) \ldots Y\left(a_{n}, z_{n}\right) \hat{1}= \\
=Y\left(\iota_{\lambda} \pi_{z_{1}}\left(e^{\lambda X}\right) a_{1}, e^{\lambda X}\left(z_{1}\right)\right) \ldots Y\left(\iota_{\lambda} \pi_{z_{n}}\left(e^{\lambda X}\right) a_{n}, e^{\lambda X}\left(z_{n}\right)\right) \hat{1}
\end{gathered}
$$

for all $X \in \mathfrak{c}_{\mathbb{C}}$ as an equality in $\mathcal{V} \llbracket z_{1} \rrbracket z_{1}^{2} \ldots \llbracket z_{n} \rrbracket z_{n}^{2} \llbracket \lambda \rrbracket$. Then in view of Eq. (8.7) and the conformal invariance of the vacuum we find

$$
\begin{gathered}
\left.\iota_{\lambda} \mathcal{W}_{n}\left(\pi_{z_{1}}\left(e^{\lambda X}\right) a_{1}, \ldots, \pi_{z_{n}}\left(e^{\lambda X}\right) a_{n} ; e^{\lambda X}\left(z_{1}\right), \ldots, e^{\lambda X}\left(z_{n}\right)\right)\right|_{\lambda=0}= \\
=\mathcal{W}_{n}\left(a_{1}, \ldots, a_{n} ; z_{1}, \ldots, z_{n}\right)
\end{gathered}
$$

for all $X \in \mathfrak{c}_{\mathbb{C}}$ which actually proves $(8.13$.

A special case of the GCI 8.13 is the translation invariance:

$$
\mathcal{W}_{n}\left(a_{1}, \ldots, a_{n} ; z_{1}, \ldots, z_{n}\right)=W_{n}\left(a_{1}, \ldots, a_{n} ; z_{12}, \ldots, z_{n-1 n}\right)
$$

$\left(z_{k l}=z_{k}-z_{l}, a_{1}, \ldots, a_{n} \in \mathcal{V}\right)$, since $\pi_{z}\left(t_{a}\right)=\mathbb{I}$. A consequence of locality for the correlation functions 8.12 is the $\mathbb{Z}_{2}$-symmetry:

$$
\mathcal{W}_{n}\left(a_{1}, \ldots, a_{n} ; z_{1}, \ldots, z_{n}\right)=(-1)^{\epsilon(\sigma)} \mathcal{W}_{n}\left(a_{\sigma(1)}, \ldots, a_{\sigma(n)} ; z_{\sigma(1)}, \ldots, z_{\sigma(n)}\right)
$$

for every permutation $\sigma \in \mathcal{S}_{n}$, where $\epsilon(\sigma)$ is the $\mathbb{Z}_{2}$-parity of $\sigma$ introduced in Proposition 4.2 
Proposition 8.2. Let $\mathcal{V}$ be a conformal vertex algebra with Hermitean structure. Then

$$
\begin{gathered}
\left(\pi_{z}(g) a\right)^{+}=\pi_{R_{D}(\bar{z})}\left(j_{W} g^{*} j_{W}^{-1}\right) a^{+} \text {or } \pi_{z}(g)^{+}=\pi_{R_{D}(\bar{z})}\left(j_{W} g^{*} j_{W}^{-1}\right) \\
\pi_{z^{* *}}\left(j_{W}\right)^{-1}\left(\pi_{z^{*}}\left(j_{W}\right)^{-1} a\right)^{+}=a
\end{gathered}
$$

Proof. As a consequence of Eqs. (8.8), (8.7) and (7.18) we have the following equalities in $\mathbb{C} \llbracket z, 1 / z^{2} \rrbracket \llbracket \lambda \rrbracket$ ( $\lambda$ being one component variable) for all $a, b, c \in \mathcal{V}$ and $X \in \mathfrak{c}_{\mathbb{C}}$ :

$$
\begin{aligned}
& \left\langle b \mid Y\left(\pi_{z}\left(e^{\lambda X}\right) a, e^{\lambda X}(z)\right) c\right\rangle=\left\langle b \mid e^{\lambda X} Y(a, z) e^{-\lambda X} c\right\rangle= \\
& =\left\langle e^{\bar{\lambda} X^{*}} Y\left(\pi_{z^{*}}\left(j_{W}\right)^{-1} a^{+}, z^{*}\right) e^{-\bar{\lambda} X^{*}} b \mid c\right\rangle= \\
& =\left\langle Y\left(\pi_{z^{*}}\left(e^{\bar{\lambda} X^{*}}\right) \pi_{z^{*}}\left(j_{W}\right)^{-1} a^{+}, e^{\bar{\lambda} X^{*}}\left(z^{*}\right)\right) b \mid c\right\rangle .
\end{aligned}
$$

On the other hand:

$$
\begin{gathered}
\left\langle b \mid Y\left(\pi_{z}\left(e^{\lambda X}\right) a, e^{\lambda X}(z)\right) c\right\rangle= \\
=\left\langle Y\left(\pi_{e^{\bar{X} X^{*}}\left(z^{*}\right)}\left(j_{W}\right)^{-1}\left(\pi_{z}\left(e^{\lambda X}\right) a\right)^{+}, e^{\bar{\lambda} X^{*}}\left(z^{*}\right)\right) b \mid c\right\rangle .
\end{gathered}
$$

Since the Hermitean form is nondegenerate, comparing the right hand sides of the last equations and replacing $g=e^{\lambda X}$ we obtain

$$
\left(\pi_{z}(g) a\right)^{+}=\pi_{g(z)^{*}}\left(j_{W}\right) \pi_{z^{*}}\left(g^{*}\right) \pi_{z^{*}}\left(j_{W}\right)^{-1} a^{+},
$$

which actually proves 8.18 if we further use the cocycle property $(7.14)$ and Eq. 8.2 .

Eq. 8.19) is a corollary of Eq. 8.18), the conjugation law $j_{W}^{*}=j_{W}^{-1}$ (see Appendix A: Eq. A.6 and the relation between $h$ and $j_{W}$ ) and the cocycle property (7.14).

\section{Connection with Globally Conformal Invariant QFT}

In this section we will construct a connection between vertex algebras and Wightman QFT with GCI in higher dimensions. For this purpose we start by introducing some additional notations. We set $D$-dimensional Minkowski space $M$ to have a signature $(D-1,1)$ so that its scalar product is:

$$
x \cdot y:=-x^{0} y^{0}+\boldsymbol{x} \cdot \boldsymbol{y} \equiv-x^{0} y^{0}+x^{1} y^{1}+\cdots+x^{D-1} y^{D-1}
$$

for $x=\left(x^{0}, \boldsymbol{x}\right) \equiv\left(x^{0}, x^{1}, \ldots, x^{D-1}\right) \in \mathbb{R}^{D}$, etc. and $x^{2} \equiv x \cdot x$. The complex Minkowski space $M_{\mathbb{C}}$ is just $M+i M \cong \mathbb{C}^{D}$ with the complexified scalar product 9.1 . For simplicity of notation we will use the same sign "." for two different scalar products (metrics) in $\mathbb{C}^{D}$ : the first is Euclidean one 1.2 used with variables labeled by letters as $z$ and $w$; the second is the above Minkowski metric used with variables labeled by letters as $x, y$ (in the real case) and $\zeta$ (in the complex case). We will identify this metrics via isomorphism:

$$
s:\left(\boldsymbol{z}, z^{D}\right) \longmapsto\left(-i z^{D}, \boldsymbol{z}\right), \quad \boldsymbol{z}:=\left(z^{1}, \ldots z^{D-1}\right) .
$$


9.1. Analytic picture on the compactified Minkowski space. The connection between the structures of the vertex algebras and QFT is based on the following coordinate transformation:

$$
\mathbb{C}^{D} \in z=\left(z, z^{D}\right) \longleftrightarrow \zeta=\left(\zeta^{0}, \zeta\right) \in \mathbb{C}^{D}
$$

where $\zeta:=\left(\zeta^{1}, \ldots, \zeta^{D-1}\right)$ and

$$
\begin{aligned}
\zeta^{0}=\frac{i}{2} \frac{1-z^{2}}{\frac{1+z^{2}}{2}+z^{D}}, & \boldsymbol{\zeta}=\frac{1}{\frac{1+z^{2}}{2}+z^{D}} \boldsymbol{z} \\
\boldsymbol{z}=\frac{1}{\frac{1+\zeta^{2}}{2}-i \zeta^{0}} \zeta, & z^{D}=\frac{1}{2} \frac{1-\zeta^{2}}{\frac{1+\zeta^{2}}{2}-i \zeta^{0}}
\end{aligned}
$$

We will regard the coordinates $\zeta$ and $z$ as two different charts on the complex compactified Minkowski space $\bar{M}_{\mathbb{C}}$. They are particular cases of the system of charts considered in Appendix A. The map

$$
h: \zeta \mapsto z,
$$

is invertible rational transformation of $\mathbb{C}^{D}$. We will use the same letter $h$ also for the transformation $s \circ h$ which can be represented by an element of the connected complex conformal group $\mathfrak{C}_{\mathbb{C}} \equiv \operatorname{Spin}_{0}(D+2 ; \mathbb{C})$ of $\mathbb{C}^{D}$ as it is derived in Appendix A. Other properties of the above transformations are:

(a) $h^{2}=j_{W}^{-1}$ (where $j_{W}$ is defined by Eq. (7.4)).

(b) If $\zeta \leftrightarrow z$ under the correspondences (9.4) and 9.5) then $\bar{\zeta} \leftrightarrow z^{*}$, where $\bar{\zeta}$ is the standard (coordinate) complex conjugation and $z^{*}$ is the conjugation given by Eq. 8.1.).

(c) The transformation $h$ is regular on $M$ and maps it on a precompact subset of $\mathbb{C}^{D}$ :

$$
\begin{gathered}
\overline{h(M)}=\bar{M}:=\left\{z \in \mathbb{C}^{D}: z^{*}=z\right\}=\left\{z \in \mathbb{C}^{D}: z=e^{i \vartheta} u\right. \\
\left.\vartheta \in \mathbb{R}, u \in \mathbb{R}^{D}, u^{2}=1\right\} \cong \mathbb{S}^{1} \times \mathbb{S}^{D-1} / \mathbb{Z}_{2}
\end{gathered}
$$

(d) Let

$$
\mathfrak{T}_{ \pm}=\left\{\xi=x+i y \in \mathbb{C}^{D}: \pm y^{0}>|\boldsymbol{y}|:=\left(\sum_{i=1}^{D-1}\left(y^{i}\right)^{2}\right)^{\frac{1}{2}}\right\}
$$

be the forward and backward tube in $\bar{M}_{\mathbb{C}}$. Then $h$ is regular on $\mathfrak{T}_{+}$and

$$
h\left(\mathfrak{T}_{+}\right)=T_{+}:=\left\{z \in \mathbb{C}^{D}:\left|z^{2}\right|<1, z \cdot \bar{z}=\left|z^{1}\right|^{2}+\cdots+\left|z^{D}\right|^{2}<\frac{1+\left|z^{2}\right|^{2}}{2}\right\} .
$$

(Note that $\mathcal{B}_{\frac{1}{2}} \varsubsetneqq T_{+} \varsubsetneqq \mathcal{B}_{1}$, where $\mathcal{B}_{\lambda}$ is the Hermitean ball $\{z: z \cdot \bar{z}<\lambda\}$.) 
These statements follow by the considerations made in Appendix A and by a straightforward computation. We will only make a comment about the derivation of Eq. 9.9. It can be obtained using the transformation properties of the interval $(\zeta-\bar{\zeta})^{2}$ under the conformal transformation $h$ (note that $\zeta \in \mathfrak{T}_{+} \cup \mathfrak{T}_{-}$iff $(\zeta-\bar{\zeta})^{2}>0$, see also [7] Eq. (A.6)). The regularity of $h$ on $\mathfrak{T}_{ \pm}$follows by the boundedness of the transformed interval $(h(\zeta)-h(\bar{\zeta}))^{2}$.

We will use the active point of view for the conformal group action. This means that the group $\mathfrak{C}_{\mathbb{C}}$ will be assumed to act on the points of the compactified Minkowski space $\bar{M}_{\mathbb{C}}$, i. e. the conformal transformations will not be considered as coordinate changes. Then every conformal transformation will have different coordinate expressions in different charts of $\bar{M}_{\mathbb{C}}$. In particular, for the above two charts the corresponding coordinate expressions for an element $g \in \mathfrak{C}_{\mathbb{C}}$ will be:

$$
\zeta \mapsto g^{(M)}(\zeta) \quad \text { and } \quad z \mapsto g(z) \quad\left(g \in \mathfrak{C}_{\mathbb{C}}\right)
$$

These two coordinate actions are connected by the conjugation $g(z)={ }_{h}^{-1}\left(g^{(M)}(h(z))\right)$. When there is no danger of confusion we will just write $g(\zeta)$ for $g^{(M)}(\zeta)$. Under these conventions we have one more property:

(e) There are two natural real forms for the complex conformal group Spin $(D+2 ; \mathbb{C})$ defined by the conjugations

$$
\begin{aligned}
& g \mapsto \bar{g}, \quad \bar{g}(z):=\overline{g(\bar{z})}, \\
& g \mapsto g^{*}, \quad g^{*}(z):=g\left(z^{*}\right)^{*}
\end{aligned}
$$

$\left(\overline{g_{1} g_{2}}=\overline{g_{1}} \overline{g_{2}}\right.$ and $\left.\left(g_{1} g_{2}\right)^{*}=g_{1}^{*} g_{2}^{*}\right)$ and the corresponding real subgroups are the Euclidean conformal group Spin $(D+1,1)$ and the Minkowski conformal group $\operatorname{Spin}(D, 2)$, respectively.

9.2. From GCI QFT to vertex algebras. We continue with the construction of the passage from the Wightman QFT with GCI to vertex algebras with Hermitean positive definite structure.

It is convenient to define the axiomatic QFT with GCI as a bilinear map $(f, a) \mapsto$ $\phi[f, a]$ which gives an operator for every complex Schwartz test function $f \in \mathfrak{S}(M)$ on Minkowski space and a vector $a$ from a complex vector space $F$. The operator $\phi[f, a]$ is supposed to act on a dense invariant domain $\mathcal{D}$ in a Hilbert space $\mathcal{H}$. The axiomatic assumptions which we impose to the fields $\phi[f, a]$ are the Wightman axioms ([4], Chapt. III) in which Lorentz covariance is replaced by the GCI condition for the correlation functions. In more details, $\phi[f, a]$ is assumed to be nonzero operator valued distribution for any fixed nonzero $a \in F$ formally written as:

$$
\phi[f, a]=\int_{M} \phi(x, a) f(x) d^{D} x .
$$

The Hermitean conjugation of the fields requires the existence of a conjugation $a \mapsto a^{+}$ on $F$ such that for all $a \in F$ and $\Psi_{1}, \Psi_{2} \in \mathcal{D}$ :

$$
\left\langle\Psi_{1} \mid \phi[f, a] \Psi_{2}\right\rangle=\left\langle\phi\left[\bar{f}, a^{+}\right] \Psi_{1} \mid \Psi_{2}\right\rangle .
$$


We demand the existence of a unitary representation of the Poincare translations which leave invariant the domain $\mathcal{D}$ and a fixed element $|0\rangle \in \mathcal{D}$ called vacuum so that they satisfy the covariance axiom, the spectral condition and the uniqueness of the vacuum. The locality axiom requires that the space $F$ is $\mathbb{Z}_{2}$-graded, $F=F_{0} \oplus F_{1}$, so that $F_{0,1}^{+}=F_{0,1}$ and

$$
\phi\left(x_{1}, a_{1}\right) \phi\left(x_{2}, a_{2}\right)-(-1)^{p_{1} p_{2}} \phi\left(x_{2}, a_{2}\right) \phi\left(x_{1}, a_{1}\right)=0
$$

if $x_{12}^{2} \equiv\left(x_{1}-x_{2}\right)^{2}>0$ and $a_{k} \in F_{p_{k}}(k=0,1)$. The condition of GCI is imposed on the correlation functions $\left\langle 0\left|\phi\left(x_{1}, a_{1}\right) \ldots \phi\left(x_{n}, a_{n}\right)\right| 0\right\rangle$ and it supposes first that there exists a cocycle $\pi_{x}^{M}(g)$ : a rational function in $x \in M$ for fixed $g \in \mathfrak{C}_{\mathbb{C}}$ with values in $(\text { End } F)_{0}$ (i. e. a ratio of a polynomial in $x$ whose coefficients are even endomorphisms of $F$ and a complex polynomial), regular in the domain of $g$ and satisfying the cocycle property (7.14) and triviality for Poincaré translations, $\pi_{x}^{M}\left(\tau_{a}\right)=\mathbb{I}_{F}\left(\tau_{a}(x):=x+a\right)$; then the correlation functions should be invariant under the substitution

$$
\phi(x, a) \mapsto \phi\left(g(x), \pi_{x}^{M}(g) a\right)
$$

in the sense of [7], Sect. 2. The cocycle should be consistent with the conjugation on $F$ in the sense that:

$$
\left[\pi_{\zeta}(g) a\right]^{+}=\pi_{\bar{\zeta}}^{M}\left(g^{*}\right) a^{+} .
$$

Note that from the triviality of $\pi_{x}^{M}\left(\tau_{a}\right)$ it follows that $\pi_{x}^{M}(g)$ does not depend on $x$ for the transformations $g$ belonging to the complex Weyl group, the complex Poincaré group with dilations, and this is an action of this group on $F$. Since the linear space $F$ can be infinite dimensional we impose an additional condition on the cocycle $\pi^{M}$ : the above representation of the complex Weyl group is supposed to be decomposable into a direct sum of finite dimensional irreducible representations. Finally, the axiom of completeness is naturally generalized. This completes our characterization of axiomatic QFT with GCI.

Under this assumptions the result about the analytic continuation of the vectorvalued distribution $\phi\left(x_{1}, a_{1}\right) \ldots \phi\left(x_{n}, a_{n}\right)|0\rangle$ ([4] IV.2) comes true: they are boundary value of the functions

$$
\Phi_{n}\left(\zeta_{1}, a_{1} ; \ldots ; \zeta_{n}, a_{n}\right) \in \mathcal{D}
$$

analytic in the tube domain

$$
\mathcal{T}_{n}:=\left\{\left(\zeta_{1}, \ldots, \zeta_{n}\right): \zeta_{k+1 k} \in \mathfrak{T}_{+} \text {for } k=1, \ldots, n-1, \zeta_{n} \in \mathfrak{T}_{+}\right\}
$$

$\left(\zeta_{k+1 k}:=\zeta_{k+1}-\zeta_{k}\right)$.

Direct generalizations of the Theorems 3.1 and 4.1 of [7] lead to rationality of the Wightman functions:

$$
\left\langle 0\left|\phi\left(x_{1}, a_{1}\right) \ldots \phi\left(x_{n}, a_{n}\right)\right| 0\right\rangle=\frac{\mathcal{P}\left(x_{1}, \ldots, x_{n} ; a_{1}, \ldots, a_{n}\right)}{\prod_{1 \leqslant k<l \leqslant n}\left(x_{k l}^{2}+i 0 x_{k l}^{0}\right)^{\mu\left(a_{k}, a_{l}\right)}},
$$

where $\mathcal{P}\left(x_{1}, \ldots, x_{n} ; a_{1}, \ldots, a_{n}\right)$ are polynomials and $\mu(a, b)$ is such a function that:

$$
\left[(x-y)^{2}\right]^{\mu(a, b)}\left[\phi(x, a) \phi(y, b)-(-1)^{p q} \phi(y, b) \phi(x, a)\right]=0
$$


for all $x, y \in M$ (in the sense of distributions) and all $a \in F_{p}$ and $b \in F_{q}(p, q=0,1)$. It is natural to expect that the rationality of the correlation functions will imply stronger analytic properties for the analytic vector functions 9.18 :

Theorem 9.1. Let the system of fields $\phi(x, a)$ satisfies the above conditions. Then the analytic vector-valued functions (9.18) possess a continuation which we will denote again by $\Phi_{n}\left(\zeta_{1}, a_{1} ; \ldots ; \zeta_{n}, a_{n}\right)$ such that it is holomorphic in the domain of all sets $\left(\zeta_{1}, \ldots, \zeta_{n}\right)$ of mutually nonisotropic points of the forward tube $\mathfrak{T}_{+}$. The functions $\left(\prod_{k<l}\left(\zeta_{k l}^{2}\right)^{\mu\left(a_{k}, a_{l}\right)}\right) \Phi_{n}\left(\zeta_{1}, a_{1} ; \ldots ; \zeta_{n}, a_{n}\right)$ possess analytic continuation in the domain of all $\zeta_{1}, \ldots, \zeta_{n} \in \mathfrak{T}_{+}$.

We will prove this theorem in Appendix B.

A straightforward corollary of Theorem 9.1 is that the real connected conformal group $\operatorname{Spin}_{0}(D, 2)$ has a unitary representation $U(g)$ on $\mathcal{H}$ such that

$U(g) \Phi_{n}\left(\zeta_{1}, a_{1} ; \ldots ; \zeta_{n}, a_{n}\right)=\Phi_{n}\left(g\left(\zeta_{1}\right), \pi_{\zeta_{1}}^{M}(g) a_{1} ; \ldots ; g\left(\zeta_{n}\right), \pi_{\zeta_{n}}^{M}(g) a_{n}\right)$

for all (Minkowski) real conformal transformations $g \in \mathfrak{C}:=\operatorname{Spin}_{0}(D, 2)$, all $\zeta_{1}$, $\ldots, \zeta_{n} \in \mathfrak{T}_{+}$for which $\zeta_{k l}^{2} \neq 0$ for $k<l$, and for all vectors $a_{1} \ldots a_{n} \in F$. Indeed, Eq. (9.22) determines $U(g)$ as a preserving norm map on a dense subspace of $\mathcal{H}$, in accord with the GCI. Note also that $\pi_{\zeta}(g)$ is defined (regular) for $g \in \operatorname{Spin}_{0}(D, 2)$ and $g(\zeta) \in \mathfrak{T}_{+}$since $\mathfrak{T}_{+}$is a homogeneous space for the real conformal group [12].

As a corollary of Theorem 9.1 the operator-valued generalized functions

$$
\left(\prod_{1 \leqslant k<l \leqslant n}\left(\zeta_{k l}^{2}\right)^{\mu\left(a_{k}, a_{l}\right)}\right) \prod_{m=1}^{n} \phi\left(x_{m}, a_{m}\right)
$$

together with all their derivatives in $x_{1}, \ldots, x_{n}$ are defined (and regular) for coinciding arguments $x_{1}=\cdots=x_{n}=: x$ and are again operator distributions in $x$ acting on a common dense invariant domain $\widetilde{\mathcal{D}} \supseteq \mathcal{D}$. Denote the linear span of all these operator functions $\psi(x)$ together with the constant function $\mathbb{I}(x) \equiv \mathbb{I}_{\mathcal{H}}$ by $\mathcal{A}$. The space $\mathcal{A}$ has natural $\mathbb{Z}_{2}$-grading induced by those of the fields $\phi(a, x)$ (i. e. the $\mathbb{Z}_{2}$-grading of $F$ ). The construction of the operator distributions $\psi(x) \in \mathcal{A}$ is done via the correlation functions (9.20) so we conclude that every correlator $\left\langle 0\left|\psi_{1}\left(x_{1}\right) \ldots \psi_{n}\left(x_{n}\right)\right| 0\right\rangle$, for $\psi_{1}, \ldots, \psi_{n} \in \mathcal{A}$, is rational and $\mathbb{Z}_{2}$-symmetric in the sense of Eq. 9.20) and the vector distribution $\psi_{1}\left(x_{1}\right) \ldots \psi_{n}\left(x_{n}\right)|0\rangle$ is a boundary value of an analytic function

$$
\mathcal{Y}_{n}^{M}\left(\psi_{1}, \zeta_{1} ; \ldots ; \psi_{n}, \zeta_{n}\right)\left(\equiv \psi_{1}\left(\zeta_{1}\right) \ldots \psi_{n}\left(\zeta_{n}\right)|0\rangle\right)
$$

satisfying Theorem 9.1 Note that

$$
\begin{aligned}
& \left\langle Y_{n}^{M}\left(\psi_{1}^{+}, \overline{\zeta_{1}} ; \ldots ; \psi_{n}^{+}, \overline{\zeta_{n}}\right) \mid Y_{m}^{M}\left(\psi_{1}^{\prime}, \zeta_{1}^{\prime} ; \ldots ; \psi_{m}^{\prime}, \zeta_{m}^{\prime}\right)\right\rangle= \\
& \quad=\left\langle 0 \mid Y_{n+m}^{M}\left(\psi_{1}, \zeta_{1} ; \ldots ; \psi_{n}, \zeta_{n} ; \psi_{1}^{\prime}, \zeta_{1}^{\prime} ; \ldots ; \psi_{m}^{\prime}, \zeta_{m}^{\prime}\right)\right\rangle
\end{aligned}
$$

(the scalar product of the vacuum with $\mathcal{Y}_{n+m}^{M}$ ) where $\psi \mapsto \psi^{+}$is the conjugation induced on $\mathcal{A}$ by the Hermitean conjugation of $\psi(x) \in \mathcal{A}$.

Next observe that the cocycle $\pi^{M}$ on $F$ gives rise to a cocycle on $\mathcal{A}$, which we denote again by $\pi^{M}$, such that it is a continuation of the initial one under the natural inclusion of $F$ in $\mathcal{A}$ and

$$
U(g) \psi(x) U(g)^{-1}=\left(\pi_{x}^{M}(g) \psi\right)(x)
$$


for $g \in \mathfrak{C}$ and $x \in M$, regular for $g$ (i. e., $g(x) \in M$ ). In particular,

$$
U(g) \phi(x, a) U(g)^{-1}=\phi\left(x, \pi_{x}^{M}(g) a\right) \quad(a \in F, \quad g(x) \in M) .
$$

Eqs. 9.25) and (9.26) are understood in the sense of distributions in the domain $\{x$ : $g(x) \in M\}$. The sketch of the arguments is the following: first observe that the action of the Weyl group on $\mathcal{A}$, which is naturally generated by those on $F$, is again decomposable into a direct sum of finite dimensional irreducible representations (since it is representable by sum of tensor products of such representations). The energy positivity (see the argument before Proposition 7.1 allows then to define an extension of the cocycle $\pi^{M}$ on $\mathcal{A}$ so that every element $\psi \in \mathcal{A}$ belongs to a finite dimensional $\pi^{M}$-invariant subspace (see Lemma 7.2). This makes sensible the action of $\pi_{x}^{M}(g)$ in Eqs. (9.25) and (9.26) as an action of multiplicators. After this precision we can prove 9.26 from $(9.22$ and $(9.25)$ is then obtained. Note also that for all $g \in \mathfrak{C}$ we have a similar equation as 9.21 for $Y_{n}^{M}\left(\psi_{1}, \zeta_{1} ; \ldots ; \psi_{n}, \zeta_{n}\right)$.

The heuristic connection between the system of fields $\mathcal{A}$ and the vertex operators $Y(a, z)$ which we are going to introduce is

$$
Y(a, z)=\left(\pi_{e(z)}^{M}(h) \psi\right)\left(h^{-1}(z)\right)
$$

where $h$ is the complex conformal transformation introduced in Sect. 9.1. In more details, let us first define the transformed vector functions

$$
\begin{gathered}
\mathscr{Y}_{n}\left(\psi_{1}, z_{1} ; \ldots ; \psi_{n}, z_{n}\right):= \\
:=Y_{n}^{M}\left(\pi_{e(z)}^{M}\left(h^{-1}\right) \psi_{1}, h^{-1}\left(z_{1}\right) ; \ldots ; \pi_{e(z)}^{M}\left(h^{-1}\right) \psi_{n}, h^{-1}\left(z_{n}\right)\right),
\end{gathered}
$$

such that the products $\left(\prod_{k<l}\left(z_{k l}^{2}\right)^{\mu_{k l}}\right) \Upsilon_{n}\left(z_{1}, a_{1} ; \ldots ; z_{n}, a_{n}\right)$ are analytic in $\left(z_{1}, \ldots\right.$, $\left.z_{n}\right) \in T_{+}^{n}$ (see Eq. (9.9) for sufficiently large $\mu_{k l} \in \mathbb{N}(1 \leqslant k<l \leqslant n)$ and they are covariant in the sense:

$$
U(g) \mathscr{Y}_{n}\left(\psi_{1}, z_{1} ; \ldots ; \psi_{n}, z_{n}\right)=\mathscr{Y}_{n}\left(\pi_{z_{1}}(g) \psi_{1}, g\left(z_{1}\right) ; \ldots ; \pi_{z_{1}}(g) \psi_{n}, g\left(z_{n}\right)\right)
$$

where

$$
\pi_{z}(g):=\pi_{e(z)}^{M}\left(h g h^{-1}\right)
$$

is a rational cocycle on $\mathcal{A}$ in the $z$-chart as those of Sect. 7 Note that both cocycles $\pi_{\zeta}^{M}(g)$ and $\pi_{z}(g)$ are constant (not depending on $\zeta$ and $z$, resp.) for different subgroups of $\mathfrak{C}_{\mathbb{C}}$ : the complex Weyl group of Minkowski space and the complex Euclidean group with dilations for $z$-chart, respectively. This is because we consider the conformal group action in an active sense. In particular, the translations $\tau_{a}$ and $t_{a}$ for both charts $\left(\tau_{a}^{(M)}(\zeta):=\zeta+a\right.$ and $\left.t_{a}(z):=z+a\right)$ are different, as in the first case these are the Poincaré translations while in the second case these translations are generated by the T's of Sect.7

Now we are ready to define the vertex algebra structure that arises from the considered GCI QFT. Set the vertex algebra space $\mathcal{V}$ to be the image of the linear map:

$$
\left.\mathcal{A} \ni \psi \mapsto و_{1}(\psi, z)\right|_{z=0} \in \mathcal{H}
$$


In fact, this map is injective as a corollary of the separating property of the vacuum (indeed, the translation covariance in 9.29 will lead to cancelation of the whole function $\mathcal{Y}_{1}(\psi, z)$ ). So we will further identify the space $\mathcal{A}$ by $\mathcal{V}$ under this injection, thus transferring the $\mathbb{Z}_{2}$-grading and the above cocycle $\pi_{z}(g)$ on $\mathcal{V}$. Note that the space $\mathcal{V}$ is dense in the Hilbert space $\mathcal{H}$ which is a consequence of the completeness axiom. The vertex algebra vacuum $\widehat{1}$ we set to be the QFT vacuum $|0\rangle$ (note that $\mathscr{Y}_{1}(\mathbb{I}, z) \equiv|0\rangle$ ). The action of the conformal Lie algebra $\mathfrak{c}_{\mathbb{C}}$ (which include the action of the translation endomorphisms $T_{\mu}$ ) is the derivative action of that of 9.29) (since $\mathcal{A}$ include all the derivative fields we will not thus leave the space $\mathcal{V}$ ). The Hermitean form on $\mathcal{V}$ is the restricted Hilbert scalar product. Finally, the state-field correspondence is defined as

$$
Y(a, z) b:=\left(z^{2}\right)^{-N} \iota\left(\left.\left[(z-w)^{2}\right]^{N} \mathscr{Y}_{2}(a, z ; b, w)\right|_{w=0}\right)
$$

for $N \gg 0$, where $\iota$ stands for the Taylor expansion at 0 of the resulting analytic function in $z$. The coefficients in the formal series 9.32 belong to $\mathcal{V}$ since the derivatives of the regularized products $\left(x_{12}^{2}\right)^{N} \psi_{1}\left(x_{1}\right) \psi_{2}\left(x_{2}\right)$ (for $N \gg 0$ ) of fields $\psi_{1}, \psi_{2} \in \mathcal{A}$, at coinciding arguments $x_{1}=x_{2}$, again belong to $\mathcal{A}$.

Theorem 9.2. The above defined $\mathcal{V}$ is a vertex algebra with Hermitean structure. The space $\mathcal{V}$ is dense in the Hilbert space $\mathcal{H}$ and coincides with the finite conformal energy space: the linear span of all eigenvectors of the conformal Hamiltonian $H$ (having a discrete spectrum).

We will sketch the proof only for some of the properties which one has to verify. First, one can find by a straightforward computation that under the transformation 9.28 the conversed Eq. 9.24 reads as

$$
\begin{gathered}
\left\langle\mathscr{Y}_{n}\left(\pi_{z_{1}^{*}}\left(j_{W}\right)^{-1} a_{1}^{+}, z_{1}^{*} ; \ldots ; \pi_{z_{n}^{*}}\left(j_{W}\right)^{-1} a_{n}^{+}, z_{n}^{*}\right) \mid \mathscr{Y}_{m}\left(b_{1}, w_{1} ; \ldots ; b_{m}, w_{m}\right)\right\rangle= \\
=\left\langle\widehat{1} \mid \mathscr{Y}_{n+m}\left(a_{1}, z_{1} ; \ldots ; a_{n}, z_{n} ; b_{1}, w_{1} ; \ldots ; b_{m}, w_{m}\right)\right\rangle
\end{gathered}
$$

(one uses Eqs. (9.17) and (A.6), the cocycle property as well as the properties (a) and (b) of the previous subsection). As a corollary of this equation and Eq. (9.32) we then obtain that Eq. 8.8 from the definition of the Hermitean structure on vertex algebra is satisfied.

Next one can derive the equality

$Y\left(a_{1}, z_{1}\right) \ldots Y\left(a_{n}, z_{n}\right) \widehat{1}=\iota_{z_{1}, \ldots, z_{n}}\left(\rho_{n}^{-N}\right) \iota\left(\rho_{n}^{N} و_{n}\left(a_{1}, z_{1} ; \ldots ; a_{n}, z_{n}\right)\right)$

for $N \gg 0$, where $\rho_{n}:=\prod_{1 \leqslant k<l \leqslant n} z_{k l}^{2}$ and the second $\iota$ stand for the Taylor expansion of the resulting analytic function in $z_{1}, \ldots, z_{n}$ at $(0, \ldots, 0)$. For this purpose, first we note that the left hand side in (9.34) belongs to $\mathcal{V} \llbracket z_{1} \rrbracket z_{1}^{2} \ldots \llbracket z_{n} \rrbracket z_{n}^{2}$ which follows inductively in $n$. Then Eq. 9.34) is proven by induction in $n$ starting from the definition equality (9.32) and for the inductive step using Eq. (9.33).

From Eq. 9.34 it follows the locality (Definition 2.1 $(b)$ ). The covariance properties of Definition 7.1 (Eqs. (7.7)-(7.9) together with Definition 2.1 (c)) follows from the conformal covariance law 9.29). Finally, we point out that the condition $(d)$ of Definition 7.1 is a consequence of the rationality of the cocycle $\pi_{z}(g)$. This condition also imply the second statement in the Theorem 9.2 
9.3. The converse passage. Here we start by a vertex algebra $\mathcal{V}$ with a strongly positive Hermitean structure. Thus $\mathcal{V}$ is a prehilbert space and denote by $\mathcal{H}$ its Hilbert completion. We are going to construct a GCI QFT on $\mathcal{H}$.

The first step is to reconstruct the functions $Y_{n}\left(a_{1}, z_{1} ; \ldots ; a_{n}, z_{n}\right)$ as an analytic in the domain of $\left(z_{1}, \ldots, z_{n}\right) \in T_{+}^{n}$. Here they are defined by the sum of the formal series $\rho_{n}^{N}\left(\prod_{m=1}^{n} Y\left(a_{m}, z_{m}\right)\right) \hat{1}$ for $N \gg 0$, where $\rho_{n}=\prod_{1 \leqslant k<l \leqslant n} z_{k l}^{2}$, divided by $\rho_{n}^{-N}$. The last formal series converges due to Fact B.3 since its Hilbert norm converges to a polynomial in accord with Proposition 8.1

Next one proves Eq. 9.33 as a consequence of the iterated Eq. 8.8 in Definition 8.1

Then one can define a unitary representation of the real conformal group $\mathfrak{C}$ on $\mathcal{H}$ via Eq. (9.29) as the right hand side does not change its Hilbert norm due to the GCI 8.13).

Now we have to make the converse passage in Eq. (9.28) and obtain the functions $\mathcal{Y}_{n}^{M}\left(a_{1}, \zeta_{1} ; \ldots ; a_{n}, \zeta_{n}\right)$ analytic in the same domain as those of previous subsection. They can be proven to satisfy again Eq. (9.24) and the corresponding conformal covariance law with a cocycle $\pi_{x}^{M}(g)$ on $\mathcal{V}$ given by the converse equality of Eq. (9.30).

Finally we define a system of local fields $\{y(a, x): a \in \mathcal{V}\}$ using the generalized vector functions obtained by the limit

$$
\mathcal{Y}_{n}^{M}\left(a_{1}, \zeta_{1} ; \ldots ; a_{n}, \zeta_{n}\right) \rightarrow y\left(a_{1}, x_{1}\right) \ldots y\left(a_{n}, x_{n}\right)|0\rangle
$$

$(|0\rangle:=\widehat{1})$, for: $\left(\zeta_{1}, \ldots, \zeta_{n}\right) \in \mathcal{T}_{+}$(Eq. (9.19)), $\operatorname{Re} \zeta_{k}=x_{k}, \operatorname{Im} \zeta_{l+1 l} \rightarrow 0(k=$ $1, \ldots, n$ and $l=1, \ldots, n-1)$ and $\operatorname{Im} \zeta_{n} \rightarrow 0$. In order to express the action of $y(a, x)$ by the vector distributions $y\left(a_{1}, x_{1}\right) \ldots y\left(a_{n}, x_{n}\right)|0\rangle$ one should use Eq. 9.24.

Summarizing the above construction we have found:

Theorem 9.3. The above defined system of fields $\{y(a, x): a \in \mathcal{V}\}$ with the cocycle $\pi_{x}^{M}(g)$ satisfies the axioms of the GCI QFT.

\section{Outlook}

In conclusion, we shall discuss some possibilities for finding new models for the vertex algebras in higher dimensions. They are based on the construction of vertex algebra from Lie algebras of formal distributions explained in Sect. [5 A straightforward generalization of the commutation relations 5.2 is presented by the so called Lie field models. In our notations these models should be defined by the relations:

$$
\begin{aligned}
{\left[u^{\alpha}\left(z_{1}\right), u^{\beta}\left(z_{2}\right)\right] } & =\sum_{\gamma=1}^{A} \frac{\int}{M} D_{\alpha \beta \gamma}\left(z_{1}, z_{2}, z_{3}\right) u^{\gamma}\left(z_{3}\right) d^{D} z_{3}+F_{\alpha \beta}\left(z_{1}, z_{2}\right) \mathbb{I} \\
D_{\alpha \beta \gamma}\left(z_{1}, z_{2}, z_{3}\right) & =\iota_{z_{3}, z_{1}, z_{2}}\left(W_{\alpha \beta \gamma}^{(3)}\left(z_{12}, z_{23}\right)\right)-\iota_{z_{3}, z_{2}, z_{1}}\left(W_{\alpha \beta \gamma}^{(3)}\left(z_{12}, z_{23}\right)\right) \\
F_{\alpha \beta}\left(z_{1}, z_{2}\right) & =\iota_{z_{1}, z_{2}}\left(W_{\alpha \beta}^{(2)}\left(z_{12}\right)\right)-\iota_{z_{2}, z_{1}}\left(W_{\alpha \beta}^{(2)}\left(z_{12}\right)\right)
\end{aligned}
$$

where $W_{\alpha \beta}^{(2)}(z, w), W_{\alpha \beta \gamma}^{(3)}(z, w) \in \mathbb{C}[z, w] z^{2} w^{2}(z+w)^{2}, z_{k l}=z_{k}-z_{l}$, and the (complex) measure $d^{D} z$ on $\bar{M}$ is provided by the restriction of the volume form $d z^{1} \wedge$ 
$\cdots \wedge d z^{D}$ on the real submanifold $\bar{M}$ (9.7). The integral of Eq. 10.1) can be understood by the substitution

$$
u^{\gamma}\left(z_{3}\right)=e^{z_{23} \cdot \partial_{z_{2}}} u^{\gamma}\left(z_{2}\right)
$$

and by formal integration of the kernel $D_{\alpha \beta \gamma}\left(z_{12}, z_{23}\right)$ with $e^{z_{23} \cdot \partial_{z_{2}}} d z_{23}$ as a series belonging to $\mathbb{C} \llbracket z_{12} \rrbracket z_{z_{12}^{2}}^{2} \llbracket \partial_{z_{2}} \rrbracket$. Sum of integrals of this type should appear in any GCI QFT with possibly infinite number $A$ of fields. It was shown by Robinson [10] that there are no nonfree (i. e. with $D_{\alpha \beta \gamma}(z, w) \neq 0$ for some $\alpha, \beta$ and $\gamma$ ) such scalar models (i. e. $A=1$ ) in space-time dimension higher than 2. For nonscalar but finite component models it is not clear whether there exists a solution of the Jacobi identity for the commutators in 10.11. From the point of view of the GCI the interesting solutions are those for which the functions $W_{\alpha \beta}^{(2)}$ and $W_{\alpha \beta \gamma}^{(3)}$ are conformally invariant (finite component) 2 - and 3-point functions. They would provide a conformal and Hermitean structure on the vertex algebra of the type considered in Sects. 7 and 8 For example there are nontrivial candidates for a tree point function of a nonabelian gauge field $F_{\mu \nu}^{a}$ displayed in [6] and it is an interesting question whether they yield the Jacobi identity for the corresponding commutators.

Acknowledgements. The author thanks Bojko Bakalov, Petko Nikolov, Ventseslav Rizov and Ivan Todorov for useful discussions. The author acknowledges the hospitality of the Erwin Schrödinger International Institute for Mathematical Physics (ESI) where this work was conceived as well as partial support by the Bulgarian National Council for Scientific Research under contract F-828, by the Research Training Network within the Framework Programme 5 of the European Commission under contract HPRN-CT-2002-00325.

\section{Appendix A. Affine system of charts on complex compactified Minkowski space}

In this section we will follow the notation of [7], Sect. 2 and Appendix A but our considerations will be basically done over the complex field $\mathbb{C}$.

Recall that as an algebraic variety, the complex $D$-dimensional compactified Minkowski space $\bar{M}_{\mathbb{C}}$ is defined as a complex $D$-dimensional projective nondegenerated quadric. The real compactified Minkowski space $\bar{M}$ is then characterized by a conjugation $\bar{M}_{\mathbb{C}} \ni p \mapsto p^{*} \in \bar{M}_{\mathbb{C}}$ as $\bar{M} \equiv\left\{p \in \bar{M}_{\mathbb{C}}: p=p^{*}\right\}$ such that the corresponding real restriction of the complex quadric has a signature $(D, 2)$.

The manifold $\bar{M}_{\mathbb{C}}$ is a homogeneous space of the connected complex conformal group $\mathfrak{C}_{\mathbb{C}} \equiv \operatorname{Spin}_{0}(D+2 ; \mathbb{C})$ which acts on it via its orthogonal action on the quadric. The stabilizer $\mathfrak{C}_{\mathbb{C}, p}$ of a point $p \in \bar{M}_{\mathbb{C}}$ is isomorphic to the complex spinor Weyl group: the complex spinor Poincaré group with dilations. The action of $\mathfrak{C}_{\mathbb{C}, p}$ leave invariant two natural subsets of $\bar{M}_{\mathbb{C}}$ : the set $K_{\mathbb{C}, p}$ of all mutually isotropic points of $\bar{M}_{\mathbb{C}}$ with respect to $p$ (recall that the isotropy relation is conformally invariant and coincides on the projective quadric with the orthogonality relation of the rays); the other invariant subspace is the complement $M_{\mathbb{C}, p}:=\bar{M}_{\mathbb{C}} \backslash K_{\mathbb{C}, p}$. The set $M_{\mathbb{C}, p}$ is open and dense in $\bar{M}_{\mathbb{C}}$ and the action of $\mathfrak{C}_{\mathbb{C}, p}$ induces on it a structure of affine space with a conformal class of flat metric. Thus we obtain a complex affine atlas $\left\{M_{\mathbb{C}, p}: p \in \bar{M}_{\mathbb{C}}\right\}$ on $\bar{M}_{\mathbb{C}}$ indexed by its points.

To illustrate the above construction note that a particular case of affine chart of $\bar{M}_{\mathbb{C}}$ is the injection of the complex Minkowski space $M_{\mathbb{C}}$ in $\bar{M}_{\mathbb{C}}, M_{\mathbb{C}} \hookrightarrow \bar{M}_{\mathbb{C}}$. In this case $M_{\mathbb{C}} \equiv M_{\mathbb{C}, p_{\infty}}$ for a special point $p_{\infty} \in \bar{M}_{\mathbb{C}}$ : the tip of the cone $K_{\infty} \equiv K_{\mathbb{C}, p_{\infty}}$ of "infinite" points. The complex spinor Weyl group of the Minkowski space $M_{\mathbb{C}}$ coincides then with $\mathfrak{C}_{\mathbb{C}, p_{\infty}}$. Every other stabilizer $\mathfrak{C}_{\mathbb{C}, p}$ is conjugated to $\mathfrak{C}_{\mathbb{C}, p_{\infty}}: \mathfrak{C}_{\mathbb{C}, p}=$ 
$g \mathfrak{C}_{\mathbb{C}, p_{\infty}} g^{-1}$ if $g\left(p_{\infty}\right)=p$. Thus $M_{\mathbb{C}, p}=g\left(M_{\mathbb{C}}\right)$ which transfers the affine structure from $M_{\mathbb{C}}$ to $M_{\mathbb{C}, p}$.

Fixing a point $q \in M_{\mathbb{C}, p}$ it becomes a vector space (with center $q$ ). In the case of the (complex) Minkowski space $M_{\mathbb{C}}$ the center is denoted by $p_{0}$ (note that $p_{0}$ and $p_{\infty}$ are real points). Thus every pair $p, q \in \bar{M}_{\mathbb{C}}$ of mutually nonisotropic points determines a vector space included in $\bar{M}_{\mathbb{C}}$ as a dense open subset. Since there always exists a transformation $g \in \mathfrak{C}_{\mathbb{C}}$ such that $g\left(p_{\infty}\right)=p$ and $g\left(p_{0}\right)=q$ ([7] Proposition 1.1) then the map $g: M_{\mathbb{C}} \rightarrow g\left(M_{\mathbb{C}}\right) \equiv M_{\mathbb{C}, p}$ will be an isomorphism of the corresponding vector spaces.

In the projective description of $\bar{M}_{\mathbb{C}}$ a vector chart $M_{\mathbb{C}, p}$ with center $q$ is determined by two representatives $\vec{\eta}_{\infty}, \vec{\eta}_{0} \in \mathbb{C}^{D+2}, p=\left\{\lambda \vec{\eta}_{\infty}\right\}, q=\left\{\lambda \vec{\eta}_{0}\right\}\left(\vec{\eta}_{\infty}^{2}=\vec{\eta}_{0}^{2}=0\right.$ as in [7] Eq. (A.1)) with fixed mutual normalization $\vec{\eta}_{\infty} \cdot \vec{\eta}_{0}=1$. Then the orthogonal complement $\left\{\vec{\eta}_{\infty}, \vec{\eta}_{0}\right\}^{\perp} \cong \mathbb{C}^{D}\left(\operatorname{Span}\left\{\vec{\eta}_{\infty}, \vec{\eta}_{0}\right\}\right.$ has nondegenerated metric) plays the role of vector space of the chart, $M_{\mathbb{C}, p} \cong\left\{\vec{\eta}_{\infty}, \vec{\eta}_{0}\right\}^{\perp}$. A particular case of the last correspondence is the Klein-Dirac compactification formulae [7] Eq. (A.2) where $\vec{\eta}_{\infty} \equiv \vec{\xi}_{\infty}$ and $\vec{\eta}_{0} \equiv \vec{\xi}_{0}$ so that

$$
\begin{gathered}
\left\{\vec{\xi}_{\infty}, \vec{\xi}_{0}\right\}^{\perp} \ni \sum_{\mu=0}^{D-1} \zeta^{\mu} \vec{e}_{\mu} \mapsto\left\{\lambda \vec{\xi}_{\zeta}\right\} \in M_{\mathbb{C}} \\
\vec{\xi}_{\zeta}:=\vec{\xi}_{0}-\frac{\zeta^{2}}{2} \vec{\xi}_{\infty}+\sum_{\mu=0}^{D-1} \zeta^{\mu} \vec{e}_{\mu}
\end{gathered}
$$

where the expressions for $\vec{\xi}_{\infty}$ and $\vec{\xi}_{0}$ can be found in Appendix A of [7]. In the general case every vector $\vec{v} \in\left\{\vec{\eta}_{\infty}, \vec{\eta}_{0}\right\}^{\perp}$ is mapped to the point $\left\{\lambda \vec{\eta}_{\vec{v}}\right\} \in M_{\mathbb{C}, p}$ determined by the representative

$$
\vec{\eta}_{\vec{v}}:=\vec{\eta}_{0}-\frac{\vec{v}^{2}}{2} \vec{\eta}_{\infty}+\vec{v} .
$$

There is a special kind of affine complex charts $M_{\mathbb{C}, p}$ determined by the condition that they cover the whole real compact Minkowski space, i. e. $\bar{M} \subset M_{\mathbb{C}, p}$ :

Proposition A.1. $\bar{M} \subset M_{\mathbb{C}, p}$ iff $p \in \mathfrak{T}_{+} \cup \mathfrak{T}_{-} \subset M_{\mathbb{C}}$ ( $\mathfrak{T}_{ \pm}$are defined in Eq. (9.8)).

Proof. First, $q \in \bar{M}$ iff $q=q^{*}$. On the after hand, let $q=q^{*}$ and $p=\{\lambda \vec{\eta}\}$, $q=\{\lambda \vec{\theta}\}$ as $\vec{\theta}=\vec{\theta}^{*}\left(\vec{\theta} \in \mathbb{R}^{D+2}\right)$. Then $q \in \bar{M}_{\mathbb{C}, p}$ iff $\vec{\eta} \cdot \vec{\theta} \neq 0$, which is also equivalent to $\vec{\eta} \cdot \vec{\theta} \neq 0$ and $\vec{\eta}^{*} \cdot \vec{\theta} \neq 0$. Thus $q \in M_{\mathbb{C}, p}$ iff $\vec{\theta} \notin \operatorname{Re}\left\{\vec{\eta}, \vec{\eta}^{*}\right\}^{\perp} \equiv$ $\{\operatorname{Re} \vec{\eta}, \operatorname{Im} \vec{\eta}\}_{\mathbb{R}}^{\perp}$ (the last one stands for orthogonal complement in $\mathbb{R}^{D+2}$ ). Therefore, $\bar{M} \subset M_{\mathbb{C}, p}$ iff the space $\{\operatorname{Re} \vec{\eta}, \operatorname{Im} \vec{\eta}\}_{\mathbb{R}}^{\perp}$ has definite restriction of the metric (i. e. it does not contain isotropic vectors). Since we have chosen the signature of $\mathbb{R}^{D+2}$ to be $(D, 2)$ we conclude that the space $\operatorname{Span}_{\mathbb{R}}\{\operatorname{Re} \vec{\eta}, \operatorname{Im} \vec{\eta}\}$ should be of signature $(0,2)$. In particular, $\vec{\eta} \cdot \vec{\xi}_{\infty} \neq 0$, i. e. $p \in M_{\mathbb{C}} \equiv M_{\mathbb{C}, p_{\infty}}$, and if we set $\vec{\eta}:=\vec{\xi}_{\zeta}$, accordingly to Eq. A.1), then we derive that $0>\left(\operatorname{Im} \vec{\xi}_{\zeta}\right)^{2}=-4\left(\vec{\xi}_{\zeta}-\vec{\xi}_{\zeta}^{*}\right)^{2}=$ $2(\operatorname{Im} \zeta)^{2}$. But the last means that $\zeta \in \mathfrak{T}_{+} \cup \mathfrak{T}_{-}$. 
Remark A.1. If we start with the real form on the quadric $\bar{M}_{\mathbb{C}}$ of signature $(r+1, s+1)$ with $s \neq 1$ and $r \neq 1$ then it follows from the above proof that there does not exist affine chart containing the whole corresponding real quadric.

Since all points of $\mathfrak{T}_{+}$as well as $\mathfrak{T}_{-}$lies in single orbits under the action of the real conformal group $\mathfrak{C}([12])$ then the charts in PropositionA.1 are conjugated one to another in both cases of $p \in \mathfrak{T}_{+}$and $p \in \mathfrak{T}_{-}$. Besides that, we prefer the case when $p \in \mathfrak{T}_{-}$because $p \notin M_{\mathbb{C}, p}$. Thus up to real conformal transformation we can choose $p$ to be the fixed point $-i e_{0}=(-i, \mathbf{0})$. The center of the chart is convenient to be the conjugated point $p^{*} \in \mathfrak{T}_{+}$since then the conjugation becomes simpler in the coordinates of the chart. The vector space of the chart is then isomorphic to $\left\{\vec{\xi}_{i e_{0}}, \vec{\xi}_{-i e_{0}}\right\}^{\perp}$ which coincides with $\operatorname{Span}\left\{\vec{e}_{1}, \ldots, \vec{e}_{D}\right\}$ in the notations of [7] Eq. (A.1). The real part of the last space is Euclidean so we denote this chart by $E_{\mathbb{C}} \hookrightarrow \bar{M}_{\mathbb{C}}, E_{\mathbb{C}} \cong \mathbb{C}^{D}$. The Eq. A.2 takes then the form similar to Eq. A.1):

$$
\mathbb{C}^{D} \ni z \mapsto\left\{\lambda \vec{\eta}_{z}\right\} \in M_{\mathbb{C}}, \quad \vec{\eta}_{z}:=\vec{\eta}_{0}-\frac{z^{2}}{2} \vec{\eta}_{\infty}+\sum_{\mu=1}^{D} z^{\mu} \vec{e}_{\mu}
$$

where $\vec{\eta}_{0}:=\frac{1}{2}\left(\vec{e}_{-1}+i \vec{e}_{0}\right)=\frac{1}{2} \vec{\xi}_{i e_{0}}$ and $\vec{\eta}_{\infty}:=-\vec{e}_{-1}+i \vec{e}_{0}=-2 \vec{\eta}_{0}^{*}$. The relations (9.4) and (9.5), giving the connection between the coordinates in the charts $M_{\mathbb{C}}$ and $E_{\mathbb{C}}$, are derived by the equation

$$
\vec{\xi}_{\zeta} \sim \vec{\eta}_{z}
$$

Note that the transformation $h$, which acts on $\mathbb{C}^{D+2}$ as an Euclidean rotation in the plain $\operatorname{Span}\left\{i \vec{e}_{0}, \vec{e}_{D}\right\}$ of an angle $\frac{\pi}{2}$, conjugates the charts $M_{\mathbb{C}}$ and $E_{\mathbb{C}}$ since

$$
h \vec{\eta}_{z}=\vec{\xi}_{s(z)}
$$

( $s$ is defined by Eq. (9.2). This is exactly the transformation (9.6) and its square acting as an Euclidean rotation in the plain $\operatorname{Span}\left\{i \vec{e}_{0}, \vec{e}_{D}\right\}$ of an angle $\pi$ is the Weyl reflection $j_{W}^{-1}$ (7.6) (here the axis of $i \vec{e}_{0}$ corresponds to the axis " $D+1$ " in the notations of Sect. [7. Note also that

$$
h^{*}=h^{-1} \text {. }
$$

We conclude this geometric review with a remark about the projective interpretation of the tube domains $\mathfrak{T}_{ \pm}\left(9.8\right.$ of the complex Minkowski space $M_{\mathbb{C}}$. First observe that for a complex vector $\vec{\eta} \in \mathbb{C}^{D+2}$ we have: $\vec{\eta}^{2}=0$ iff $(\operatorname{Re} \vec{\eta})^{2}=(\operatorname{Im} \vec{\eta})^{2}$ and $(\operatorname{Re} \vec{\eta}) \cdot(\operatorname{Im} \vec{\eta})=0$. Therefore, there are three natural subsets of rays $\{\lambda \vec{\eta}\} \in \bar{M}_{\mathbb{C}}$, invariant with respect to the real conformal group $\mathfrak{C}$ :

(a) $(\operatorname{Re} \vec{\eta})^{2}=(\operatorname{Im} \vec{\eta})^{2}>0$,

(b) $(\operatorname{Re} \vec{\eta})^{2}=(\operatorname{Im} \vec{\eta})^{2}<0$,

(c) $(\operatorname{Re} \vec{\eta})^{2}=(\operatorname{Im} \vec{\eta})^{2}=0$. 
In the selected signature $(D, 2)$ of the real quadric $\bar{M}$, the case $(b)$ corresponds to the union $\mathfrak{T}_{+} \cup \mathfrak{T}_{-}$: this follows from the equality: $\left(\operatorname{Im} \vec{\xi}_{\zeta}\right)^{2}=2(\operatorname{Im} \zeta)^{2}$, for $\zeta \in M_{C}$, already used in the proof of Proposition A.1 The separation between $\mathfrak{T}_{+}$and $\mathfrak{T}_{-}$in the subspace $(b)$ of rays, corresponds to the orientation of the pair $(\operatorname{Re} \vec{\eta}, \operatorname{Im} \vec{\eta})$.

\section{Appendix B. Proof of Theorem 9.1}

To prove the theorem 9.1 we need some technical preparation.

Proposition B.2. Let $\Theta_{0}\left(\zeta_{1}, \ldots, \zeta_{n}\right)$ be a continuous function with values in a Hilbert space $\mathcal{H}$ and defined in an open domain $U_{0}$ in $M_{\mathbb{C}}^{n}$. Let $U_{0}$ be contained in a connected domain $U$ in $M_{\mathbb{C}}^{n}$, such that the scalar product

$$
F_{0}\left(\zeta_{1}, \ldots, \zeta_{2 n}\right):=\left\langle\Theta_{0}\left(\overline{\zeta_{1}}, \ldots, \overline{\zeta_{n}}\right) \mid \Theta_{0}\left(\zeta_{n+1}, \ldots, \zeta_{2 n}\right)\right\rangle
$$

$\overline{(\zeta} \equiv \overline{x+i y}=x-i y$ for $x, y \in M)$ has a continuation to a strongly analytic function $F\left(\zeta_{1}, \ldots, \zeta_{2 n}\right)$ in the domain $U^{*} \times U$ in $M_{\mathbb{C}}^{\times 2 n}$, where $U^{*}$ is the set of complex conjugate elements of $U$. Then there exists a single-valued continuation $\Theta\left(\zeta_{1}, \ldots, \zeta_{n}\right)$ of $\Theta_{0}\left(\zeta_{1}, \ldots, \zeta_{n}\right)$, to strongly analytic function (i. e. analytic in norm) on the domain $U$, with values in $\mathcal{H}$ such that

$$
F\left(\zeta_{1}, \ldots, \zeta_{2 n}\right)=\left\langle\Theta\left(\overline{\zeta_{1}}, \ldots, \overline{\zeta_{n}}\right) \mid \Theta\left(\zeta_{n+1}, \ldots, \zeta_{2 n}\right)\right\rangle
$$

in $U^{*} \times U$.

To prove this proposition it is useful the following simple fact:

Fact B.3. Let $\left\{\Psi_{n}\right\}_{n=1}^{\infty} \subset \mathcal{H}$, then if the sequence $\left\{\left\langle\Psi_{m} \mid \Psi_{n}\right\rangle\right\}_{m, n=1}^{\infty}$ is Cauchy fundamental then the sequence $\left\{\Psi_{n}\right\}_{n=1}^{\infty}$ is fundamental in the norm of $\mathcal{H}$ too.

This follows from $\left\|\Psi_{m}-\Psi_{n}\right\|^{2}=\left\langle\Psi_{m} \mid \Theta_{m}\right\rangle+\left\langle\Psi_{n} \mid \Psi_{n}\right\rangle-2\left\langle\Psi_{m} \mid \Psi_{n}\right\rangle_{m, n \rightarrow \infty}^{\longrightarrow} 0$. Continuing with the proof of Proposition B.2 we first observe that the vector-valued function $\Theta_{0}\left(\zeta_{1}, \ldots, \zeta_{n}\right)$ is strongly analytical in the domain $U_{0}$, which follows from the analyticity of the scalar product $\mathrm{B.1}$ and the above Fact B.3 Let $\left(\zeta_{1}, \ldots, \zeta_{n}\right) \in$ $U$. The domain $U$ is connected and consequently there exists piecewise linear path in $U$ connecting $\left(\zeta_{1}, \ldots, \zeta_{n}\right)$ with $U_{0}$. Then for every interval $\left(\zeta_{1}(t), \ldots, \zeta_{n}(t)\right)$, $t \in[0,1]$ we can make the requested continuation by the following fact:

If $\Psi_{0}(t)$ is a strongly analytic function with values in $\mathcal{H}$ defined in a neighborhood of $t=0 \in \mathbb{C}$ and such that the scalar product $h_{0}\left(t_{1}, t_{2}\right):=\left\langle\Psi_{0}\left(\overline{t_{1}}\right) \mid \Psi_{0}\left(t_{2}\right)\right\rangle$ possess an analytic continuation $h\left(t_{1}, t_{2}\right)$ in $\left(t_{1}, t_{2}\right)$ in some neighborhood of $[0,1]^{\times 2} \subset \mathbb{C}^{2}$, then the function $\Psi_{0}(t)$ can be continued in a neighborhood of $[0,1]$ in $\mathbb{C}$ to strongly analytic function $\Psi(x)$, for which $h\left(t_{1}, t_{2}\right)=\left\langle\Psi\left(\overline{t_{1}}\right) \mid \Psi\left(t_{2}\right)\right\rangle$.

To prove the last statement we first note that there exists a positive number $\rho$ such that if $D_{\rho} \subset \mathbb{C}$ stands for the open complex disk with centre at $0 \in \mathbb{C}$ and radius $\rho$, then the set $[0,1]^{\times 2}+D_{\rho}^{\times 2}=\left\{u+v: u \in[0,1]^{\times 2}, v \in D_{\rho}^{\times 2}\right\}$ is contained in the analyticity domain of $h\left(t_{1}, t_{2}\right)$. Thus, if for every $t \in[0,1], J_{t}$ stands for the interval in $\mathbb{R}$ with centre $t$ and radius $\rho$, then $J_{t} \times J_{t}$ lies in the convergence domain 
of the Taylor expansion of the function $h\left(t_{1}, t_{2}\right)$ around $(t, t)$. Since the length of the intervals $J_{t}$ is constant, then there exist a finite number $J_{t_{k}}$, for $k=0, \ldots, n$ such that $0=t_{0}<\cdots<t_{k}<\cdots<t_{n}=1$ and $t_{k+1} \in J_{t_{k}}(0 \leqslant k<n)$. In such a way if the requested analytic expansion $\Psi(t)$ is done in a neighborhood of $\left[0, t_{k}\right]$ $(0 \leqslant k \leqslant n)$, then the Taylor expansion of $\Psi(t)$ with centre $t_{k}$ will be convergent in $J_{k}$ - by the construction of $J_{k}$ and Fact B.3 Thus we arrive to $t_{k+1}$ and by induction, to $t_{n}=1$.

The single-vauledness of the obtained continuation $\Theta\left(\zeta_{1}, \ldots, \zeta_{n}\right)$ follows by Eq. (B.2). Indeed, if we denote by $\mathcal{H}_{0}$, the closed linear span of the vectors of the type $\Theta_{0}\left(\zeta_{1}, \ldots, \zeta_{n}\right)$, for all $\left(\zeta_{1}, \ldots, \zeta_{n}\right)$ in $U_{0}$, then in accord with the above construction the continuation $\Theta\left(\zeta_{1}, \ldots, \zeta_{n}\right)$ will take its values again in $\mathcal{H}_{0}$. Thus the singlevaluedness of $\Theta\left(\zeta_{1}, \ldots, \zeta_{1}\right)$ will follow by the single-valuedness of the scalar product (B.2). The strong analyticity of $\Theta\left(\zeta_{1}, \ldots, \zeta_{1}\right)$ is implied again by Eq. B.2. as those of $\Theta_{0}\left(\zeta_{1}, \ldots, \zeta_{n}\right)$.

To prove the Theorem 9.1 we just need to apply the Proposition B.2 for the analytic function (9.18), taking $U_{0}$ to be $\mathcal{T}_{n}$ (9.19) and for $U$ using the domain of all sets of mutually nonisotropic points of $\mathfrak{T}_{+}$.

\section{References}

1. R. E. Borcherds, Vertex Algebras, q-qlg/9706008

2. B. A. Dubrovin, S. P. Novikov, A. T. Fomenko, Modern Geometry: Methods and Applications, 1992

3. R. Haag, Local Quantum Physics: Fields, Particles, Algebras, 2nd revised edition Springer Verlag (1996)

4. R. Jost, The General Theory of Quantized Fields, Amer. Math. Soc. Publ., Providence R.I. 1965

5. V. Kac, Vertex Algebras for Beginners, AMS, Providence, R. I. 1996

6. N. M. Nikolov, Ya. S. Stanev, I. T. Todorov, Globally conformal invariant gauge field theory with rational correlation functions, hep-th/0305200

7. N. M. Nikolov, I. T. Todorov, Rationality of Conformally Invariant Local Correlation Functions on Compactified Minkowski Space, Commun. Math. Phys. 218 (2001) 417-436, hep-th/0009004

8. N. M. Nikolov, I. T. Todorov, Conformal Quantum Field Theory in Two and Four Dimensions, in: Proceedings of the Summer School in Modern Mathematical Physics, eds. B. Dragovich, B. Sazdović, Belgrade 2002, pp. 1-49

9. N. M. Nikolov, I. T. Todorov, Finite Temperature Correlation Functions and Modular Forms in a Globally Conformal Invariant QFT, in preparation

10. D. W. Robinson, On a soluble model of relativistic field theory, Phys. Lett. 9 (1964) 189-191

11. I. T. Todorov, Infinite dimensional Lie algebras in conformal QFT models, in: Conformal Groups and Related Symmetries, Lecture Notes in Physics 261, ads. A. O. Barut, H.-D. Doebner, Springer, Berlin at al. 1986, pp. 387-443

12. A. Uhlmann, Remarks on the future tube, Acta Phys, Pol. 24, (1963) 293; The closure of Minkowski space, ibid. pp. 295-296; Some properties of the future tube, preprint KMU-HEP 7209 (Leipzig, 1972) 